\title{
Gevrey regularity and analyticity for Camassa-Holm type
}

\section{systems}

\author{
Wei Luo $^{1 *}$ and Zhaoyang Yin ${ }^{1,2 \dagger}$ \\ ${ }^{1}$ Department of Mathematics, Sun Yat-sen University, \\ Guangzhou, 510275, China \\ ${ }^{2}$ Faculty of Information Technology, \\ Macau University of Science and Technology, Macau, China
}

\begin{abstract}
In this paper we mainly investigate the Cauchy problem of some Camassa-Holm type systems. By constructing a new auxiliary function, we present a generalized Ovsyannikov theorem. By using this theorem and the basic properties of Sobolev-Gevrey spaces, we prove the Gevrey regularity and analyticity of these systems. Moreover, we obtain a lower bound of the lifespan and the continuity of the data-to-solution map.
\end{abstract}

2010 Mathematics Subject Classification: 35 Q53 (35B30 35B44 35C07 35G25)

Keywords: Camassa-Holm type systems; a generalized Ovsyannikov theorem; Gevrey regularity; analyticity.

\section{Contents}

\section{Introduction}

*E-mail: luowei23@mail2.sysu.edu.cn

†E-mail: mcsyzy@mail.sysu.edu.cn 
3 A generalized Ovsyannikov theorem

4 Gevrey regularity and analyticity

5 Continuity of the data-to-solution map

\section{Introduction}

In this paper we mainly consider the Cauchy problem for some Camassa-Holm type systems which can be rewritten in the following abstract form:

$$
\left\{\begin{array}{l}
\frac{d u}{d t}=F(t, u(t)), \\
\left.u\right|_{t=0}=u_{0} .
\end{array}\right.
$$

In the following, we will prove the well-posedness of (1.1) in Sobolev-Gevrey spaces under some suitable conditions on the function $F$. The most important and famous equation in (1.1) is the Camassa-Holm equation $(\mathrm{CH})$ :

$$
\left\{\begin{array}{l}
m_{t}+2 m_{x} u+m u_{x}=0, \quad m=u-u_{x x} \\
\left.m\right|_{t=0}=m_{0}
\end{array}\right.
$$

or equivalently

$$
\left\{\begin{array}{l}
u_{t}=-u \partial_{x}-\partial_{x}\left(1-\partial_{x x}\right)^{-1}\left[u^{2}+\frac{1}{2}\left(u_{x}\right)^{2}\right], \\
\left.u\right|_{t=0}=u_{0} .
\end{array}\right.
$$

The Camassa-Holm equation was derived as a model for shallow water waves [6, 17. It has been investigated extensively because of its great physical significance in the past two decades. The $\mathrm{CH}$ equation has a bi-Hamiltonian structure [8, 24] and is completely integrable [6, 9]. The solitary wave solutions of the $\mathrm{CH}$ equation were considered in [6, 7, where the authors showed that the $\mathrm{CH}$ equation possesses peakon solutions of the form $C e^{-|x-C t|}$. It is worth mentioning that the peakons are solitons and their shape is alike that of the travelling water waves of greatest height, arising as solutions to the free-boundary problem for incompressible Euler equations over a flat bed (these being the governing equations for water waves), cf. the discussions in [11, 15, 16, 46. Constantin and Strauss verified that the peakon solutions of the $\mathrm{CH}$ equation are orbitally stable in [19].

The local well-posedness for the $\mathrm{CH}$ equation was studied in [12, 13, 21, 41. Concretely, for

initial profile $u_{0} \in H^{s}(\mathbb{R})$ with $s>\frac{3}{2}$, it was shown in [12, 13, 41] that the $\mathrm{CH}$ equation has a unique solution in $C\left([0, T) ; H^{s}(\mathbb{R})\right)$. Moveover, the local well-posedness for the CH equation in Besov spaces 
$C\left([0, T) ; B_{p, r}^{s}(\mathbb{R})\right)$ with $s>\max \left(\frac{3}{2}, 1+\frac{1}{p}\right)$ was proved in [21]. The global existence of strong solutions was established in [10, 12, 13] under some sign conditions and it was shown in [10, 12, 13, 14, that the solutions will blow up in finite time when the slope of initial data was bounded by a negative quantity. The global weak solutions for the $\mathrm{CH}$ equation were studied in [18] and [47. The global conservative and dissipative solutions of $\mathrm{CH}$ equation were presented in [4] and [5], respectively. The analyticity for the solutions of $\mathrm{CH}$ equation were investigated in [3] and [32].

A natural idea is to extend such study to the multi-component generalized systems. One of the most popular generalized systems is the following integrable two-component Camassa-Holm shallow water system $(2 \mathrm{CH})[20$ :

$$
\left\{\begin{array}{l}
m_{t}+u m_{x}+2 u_{x} m+k \rho \rho_{x}=0, \quad m=u-u_{x x} \\
\rho_{t}+(u \rho)_{x}=0 \\
\left.m\right|_{t=0}=m_{0},\left.\rho\right|_{t=0}=\rho_{0}
\end{array}\right.
$$

or equivalently

$$
\left\{\begin{array}{l}
u_{t}=-u \partial_{x}-\partial_{x}\left(1-\partial_{x x}\right)^{-1}\left[u^{2}+\frac{1}{2}\left(u_{x}\right)^{2}+\frac{k}{2} \rho^{2}\right], \\
\rho_{t}=-(u \rho)_{x}, \\
\left.u\right|_{t=0}=u_{0},\left.\rho\right|_{t=0}=\rho_{0},
\end{array}\right.
$$

where $k= \pm 1$. Local well-posedness for $(2 \mathrm{CH})$ with the initial data in Sobolev spaces and in Besov spaces was established in [20, 22], and [30, respectively. The blow-up phenomena and global existence of strong solutions to $(2 \mathrm{CH})$ in Sobolev spaces were obtained in [22, 25] and [30]. The existence of global weak solutions for $(2 \mathrm{CH})$ with $k=1$ was investigated in [27.

Another one is the modified two-component Camassa-Holm system (M2CH) 31]:

$$
\left\{\begin{array}{l}
m_{t}+u m_{x}+2 u_{x} m+k \rho \bar{\rho}_{x}=0, \quad m=u-u_{x x} \\
\rho_{t}+(u \rho)_{x}=0, \quad \rho=\left(1-\partial_{x}^{2}\right)\left(\bar{\rho}-\bar{\rho}_{0}\right) \\
\left.m\right|_{t=0}=u_{0},\left.\rho\right|_{t=0}=\rho_{0}
\end{array}\right.
$$

or equivalently

$$
\left\{\begin{array}{l}
u_{t}=-u \partial_{x}-\partial_{x}\left(1-\partial_{x x}\right)^{-1}\left[u^{2}+\frac{1}{2}\left(u_{x}\right)^{2}+\frac{k}{2} \gamma^{2}-\frac{k}{2} \gamma_{x}^{2}\right] \\
\gamma_{t}=-u \gamma_{x}-\left(1-\partial_{x x}\right)^{-1}\left(\left(u_{x} \gamma_{x}\right)_{x}+u_{x} \gamma\right) \\
\left.u\right|_{t=0}=u_{0},\left.\gamma\right|_{t=0}=\gamma_{0}
\end{array}\right.
$$

where $k= \pm 1$ and $\bar{\rho}_{0}$ is a constant. Local well-posedness for $(\mathrm{M} 2 \mathrm{CH})$ with the initial data in Sobolev spaces and in Besov spaces was established in 26] and [49] respectively. The blow up phenomena of 
strong solutions to $(\mathrm{M} 2 \mathrm{CH})$ were presented in [26]. The existence of global weak solutions for (M2CH) with $k=1$ was investigated in [28. The global conservative and dissipative solutions of (M2CH) equation were studied in 42 and 43 , respectively. The analyticity of the solutions for (M2CH) was proved in 48 .

Recently Geng and Xue proposed a new three-component Camassa-Holm system with N-peakon solutions [29]:

$$
\left\{\begin{array}{l}
u_{t}=-v a_{x}+u_{x} b+\frac{3}{2} u b_{x}-\frac{3}{2} u\left(a_{x} c_{x}-a c\right), \\
v_{t}=2 v b_{x}+v_{x} b, \\
w_{t}=-v c_{x}+w_{x} b+\frac{3}{2} w b_{x}+\frac{3}{2} w\left(a_{x} c_{x}-a c\right), \\
u=a-a_{x x}, \\
v=\frac{1}{2}\left(b_{x x}-4 b+a_{x x} c_{x}-c_{x x} a_{x}+3 a_{x} c-3 a c_{x}\right), \\
w=c-c_{x x}, \\
\left.u\right|_{t=0}=u_{0},\left.\quad v\right|_{t=0}=v_{0},\left.\quad w\right|_{t=0}=w_{0} .
\end{array}\right.
$$

It is based on the following spectral problem

$$
\phi_{x}=U \phi, \quad \phi=\left(\begin{array}{l}
\phi_{1} \\
\phi_{2} \\
\phi_{3}
\end{array}\right), \quad U=\left(\begin{array}{ccc}
0 & 1 & 0 \\
1+\lambda v & 0 & u \\
\lambda w & 0 & 0
\end{array}\right),
$$

where $u, v, w$ are three potentials and $\lambda$ is a constant spectral parameter. It was shown in [29] that the N-peakon solitons of the system (1.1) have the form

$$
\begin{aligned}
& a(t, x)=\sum_{i=0}^{N} a_{i}(t) e^{-\left|x-x_{i}(t)\right|}, \\
& b(t, x)=\sum_{i=0}^{N} b_{i}(t) e^{-2\left|x-x_{i}(t)\right|}, \\
& c(t, x)=\sum_{i=0}^{N} c_{i}(t) e^{-\left|x-x_{i}(t)\right|},
\end{aligned}
$$

where $a_{i}, b_{i}, c_{i}$ and $x_{i}$ evolve according to a dynamical system. Moreover, the author derived infinitely many conservation laws of the system (1.1). In [35, the authors proved the local well-posedness and global existence of strong solution to $(3 \mathrm{CH})$ under some sign conditions.

Many researchers have studied the analyticity of solutions to Camassa-Holm type systems, cf. [3], 32 and [48. However, to our best acknowledge, the Gevrey regularity of solutions to the Camassa- 
Holm equation is still an open problem. Our motivation is to solve this problem. To begin with, we introduce an abstract Cauchy-Kovalevsky theorem which is very crucial to study the analyticity:

Theorem 1.1. [1, 36, 38] Let $\left\{X_{\delta}\right\}_{0<\delta<1}$ be a scale of decreasing Banach spaces, namely, for any $\delta^{\prime}<\delta$ we have $X_{\delta} \subset X_{\delta^{\prime}}$ and $\|\cdot\|_{\delta^{\prime}} \leq\|\cdot\|_{\delta}$, and let $T, R>0, \sigma \geq 1$. For given $u_{0} \in X_{1}$, assume that the function $F$ satisfies the following conditions:

(1) If for $0<\delta^{\prime}<\delta<1$ the function $t \mapsto u(t)$ is holomorphic in $|t|<T$ and continuous on $|t|<T$ with values in $X_{s}$ and

$$
\sup _{|t|<T}\|u(t)\|_{\delta}<R
$$

then $t \mapsto F(t, u(t))$ is a holomorphic function on $|t|<T$ with values in $X_{\delta^{\prime}}$.

(2) For any $0<\delta^{\prime}<\delta<1$ and any $u, v \in \overline{B\left(u_{0}, R\right)} \subset X_{\delta}$, there exists a positive constant $L$ depending on $u_{0}$ and $R$ such that

$$
\sup _{|t|<T}\|F(t, u)-F(t, v)\|_{\delta^{\prime}} \leq \frac{L}{\delta-\delta^{\prime}}\|u-v\|_{\delta}
$$

(3) For any $0<\delta<1$, there exists a positive constant $M$ depending on $u_{0}$ and $R$ such that

$$
\sup _{|t|<T}\left\|F\left(t, u_{0}\right)\right\|_{\delta} \leq \frac{M}{1-\delta} .
$$

Then there exists a $T_{0} \in(0, T)$ and a unique solution to the Cauchy problem (1.1), which for every $\delta \in(0,1)$ is holomorphic in $|t|<T_{0}(1-\delta)$ with values in $X_{\delta}$.

Theorem 1.1 was first proposed by Ovsyannikov in [38, [39, [40]. However, the original Ovsyannikov theorem becomes invalid for the Gevrey class. Because this kind of spaces do not satisfy the condition (2) of the Ovsyannikov theorem. More precisely, in Section 2, for the Gevrey class, we see that

$$
\sup _{|t|<T}\|F(t, u)-F(t, v)\|_{\delta^{\prime}} \leq \frac{L}{\left(\delta-\delta^{\prime}\right)^{\sigma}}\|u-v\|_{\delta},
$$

with $\sigma \geq 1$. If $\sigma>1$, the inequality (1.4) is weaker than the condition (2) because it is nonlinear decay. Thus, we need a new framework which is associated with the properties of the Gevrey class. In this paper, we modify the proof of [38] and establish a new auxiliary function, then obtain a generalised Ovsyannikov theorem. By using this theorem, we obtain both the Gevrey regularity and analyticity of the solutions to Camassa-Holm type systems. Moreover, by taking advantage of the idea in [3], we prove that the continuity of the data-to-solution map.

The paper is organized as follows. In Section 2 we recall some properties about Sobolev-Gevrey spaces. In Section 3, we prove a generalized Ovsyannikov theorem. In Section 4, we prove the analyticity and Gevrey regularity of the solutions to some Camassa-Holm type systems. In Section 5, we show that the data-to-solution map is continuous from the data space to the solution space. 


\section{Preliminaries}

Firstly, we introduce the Sobolev-Gevrey spaces and recall some basic properties.

Definition 2.1. 203] Let $s$ be a real number and $\sigma, \delta>0$. A function $f \in G_{\sigma, s}^{\delta}\left(\mathbb{R}^{d}\right)$ if and only if $f \in C^{\infty}\left(\mathbb{R}^{d}\right)$ and satisfies

$$
\|f\|_{G_{\sigma, s}^{\delta}\left(\mathbb{R}^{d}\right)}=\left(\int_{\mathbb{R}^{d}}\left(1+|\xi|^{2}\right)^{s} e^{2 \delta|\xi| \frac{1}{\sigma}}|\widehat{f}(\xi)|^{2} d \xi\right)^{\frac{1}{2}}<\infty .
$$

Remark 2.2. Denoting the Fourier multiplier $e^{\delta(-\Delta)^{\frac{1}{2 \sigma}}}$ by

$$
e^{\delta(-\Delta)^{\frac{1}{2 \sigma}}} f=\mathscr{F}^{-1}\left(e^{\delta|\xi| \frac{1}{\sigma}} \widehat{f}\right)
$$

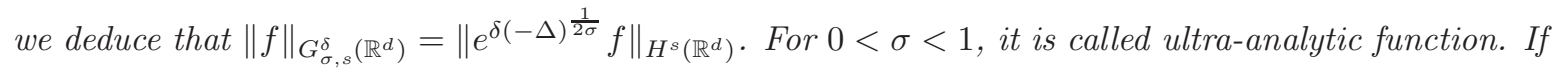
$\sigma=1$, it is usual analytic function and $\delta$ is called the radius of analyticity. If $\sigma>1$, it is the Gevrey class function.

Proposition 2.3. Let $0<\delta^{\prime}<\delta, 0<\sigma^{\prime}<\sigma$ and $s^{\prime}<s$. From Definition 2.1, one can check that $G_{\sigma, s}^{\delta}\left(\mathbb{R}^{d}\right) \hookrightarrow G_{\sigma, s}^{\delta^{\prime}}\left(\mathbb{R}^{d}\right), G_{\sigma^{\prime}, s}^{\delta}\left(\mathbb{R}^{d}\right) \hookrightarrow G_{\sigma, s}^{\delta}\left(\mathbb{R}^{d}\right)$ and $G_{\sigma, s}^{\delta}\left(\mathbb{R}^{d}\right) \hookrightarrow G_{\sigma, s^{\prime}}^{\delta}\left(\mathbb{R}^{d}\right)$.

Proposition 2.4. Let $s$ be a real number and $\sigma>0$. Assume that $0<\delta^{\prime}<\delta$. Then we have

$$
\left\|\partial_{x} f\right\|_{G_{\sigma, s}^{\delta^{\prime}(\mathbb{R})}} \leq \frac{e^{-\sigma} \sigma^{\sigma}}{\left(\delta-\delta^{\prime}\right)^{\sigma}}\|f\|_{G_{\sigma, s}^{\delta}(\mathbb{R})}
$$

Proof. Since $\widehat{\partial_{x} f}=i \xi \widehat{f}$, it follows that

$$
\begin{aligned}
\left\|\partial_{x} f\right\|_{G_{\sigma, s}^{\delta^{\prime}}(\mathbb{R})}^{2} & =\int_{\mathbb{R}}\left(1+|\xi|^{2}\right)^{s} e^{2 \delta^{\prime}|\xi|^{\frac{1}{\sigma}}}|\xi|^{2}|\widehat{f}(\xi)|^{2} d \xi \\
& =\frac{1}{\left(\delta-\delta^{\prime}\right)^{2 \sigma}} \int_{\mathbb{R}}\left(1+|\xi|^{2}\right)^{s} e^{2 \delta|\xi|^{\frac{1}{\sigma}}} e^{-2\left[\left(\delta-\delta^{\prime}\right)^{\sigma}|\xi|\right]^{\frac{1}{\sigma}}}\left(\delta-\delta^{\prime}\right)^{2 \sigma}|\xi|^{2}|\widehat{f}(\xi)|^{2} d \xi \\
& \leq \frac{\|f\|_{G_{\sigma, s}^{\delta}(\mathbb{R})}^{2}}{\left(\delta-\delta^{\prime}\right)^{2 \sigma}} \sup _{\xi \in \mathbb{R}}\left\{e^{-2\left[\left(\delta-\delta^{\prime}\right)^{\sigma}|\xi|\right]^{\frac{1}{\sigma}}}\left(\delta-\delta^{\prime}\right)^{2 \sigma}|\xi|^{2}\right\} .
\end{aligned}
$$

Let $z=\left[\left(\delta-\delta^{\prime}\right)^{\sigma}|\xi|\right]^{\frac{1}{\sigma}} \geq 0$ and consider the function $g(z)=e^{-2 z} z^{2 \sigma}$. By directly calculating, we have $\lim _{z \rightarrow 0} g(z)=0, \lim _{z \rightarrow+\infty} g(z)=0$ and $g^{\prime}(z)=-2 e^{-2 z} z^{2 \sigma}+2 \sigma e^{-2 z} z^{2 \sigma-1}$. By solving $g^{\prime}(z)=0$, we obtain that $z=\sigma$, which implies that $g(z) \leq g(\sigma)=e^{-2 \sigma} \sigma^{2 \sigma}$. Then, we deduce from (2.1) that

$$
\left\|\partial_{x} f\right\|_{G_{\sigma, s}^{\delta^{\prime}(}(\mathbb{R})} \leq \frac{e^{-\sigma} \sigma^{\sigma}\|f\|_{G_{\sigma, s}^{\delta}(\mathbb{R})}}{\left(\delta-\delta^{\prime}\right)^{\sigma}}
$$


Proposition 2.5. (Product acts on Sobolev-Gevrey spaces with $d=1$ ) Let $s>\frac{1}{2}, \sigma \geq 1$ and $\delta>0$. Then, $G_{\sigma, s}^{\delta}(\mathbb{R})$ is an algebra. Moreover, there exists a constant $C_{s}$ such that

$$
\|f g\|_{G_{\sigma, s}^{\delta}(\mathbb{R})} \leq C_{s}\|f\|_{G_{\sigma, s}^{\delta}(\mathbb{R})}\|g\|_{G_{\sigma, s}^{\delta}(\mathbb{R})} .
$$

Proof. Since $\widehat{f g}=\widehat{f} * \widehat{g}$, it follows that

$$
\begin{aligned}
& \|f g\|_{G_{\sigma, s}^{\delta}(\mathbb{R})}^{2}=\int_{\mathbb{R}}\left(1+|\xi|^{2}\right)^{s} e^{2 \delta|\xi|^{\frac{1}{\sigma}}}|\widehat{f} * \widehat{g}|^{2} d \xi \\
& =\int_{\mathbb{R}}\left(1+|\xi|^{2}\right)^{s}\left(\int_{\mathbb{R}} e^{\delta|\xi| \frac{1}{\sigma}} \widehat{f}(\eta) \widehat{g}(\xi-\eta) d \eta\right)^{2} d \xi \\
& \leq \int_{\mathbb{R}}\left(1+|\xi|^{2}\right)^{s}\left(\int_{\mathbb{R}} e^{\delta|\xi-\eta|^{\frac{1}{\sigma}}} e^{\delta|\eta|^{\frac{1}{\sigma}}} \widehat{f}(\eta) \widehat{g}(\xi-\eta) d \eta\right)^{2} d \xi \quad \text { (Here we use the fact that } \sigma \geq 1 \text { ) } \\
& =\int_{\mathbb{R}}\left(1+|\xi|^{2}\right)^{s}\left|\mathscr{F}\left(e^{\delta(-\Delta) \frac{1}{2 \sigma}} f\right) * \mathscr{F}\left(e^{\delta(-\Delta)^{\frac{1}{2 \sigma}}} g\right)\right|^{2} d \xi=\left\|\left(e^{\delta(-\Delta)^{\frac{1}{2 \sigma}}} f\right) \cdot\left(e^{\delta(-\Delta)^{\frac{1}{2 \sigma}}} g\right)\right\|_{H^{s}(\mathbb{R})}^{2} \\
& \leq C_{s}\left\|e^{\delta(-\Delta)^{\frac{1}{2 \sigma}}} f\right\|_{H^{s}(\mathbb{R})}^{2}\left\|e^{\delta(-\Delta)^{\frac{1}{2 \sigma}}} g\right\|_{H^{s}(\mathbb{R})}^{2} \quad \text { (Here we use the fact that } s>\frac{1}{2} \text { ) } \\
& =C_{s}\|f\|_{G_{\sigma, s}^{\delta}(\mathbb{R})}^{2}\|g\|_{G_{\sigma, s}^{\delta}(\mathbb{R})}^{2} .
\end{aligned}
$$

Proposition 2.6. Let $s>\frac{1}{2}, \sigma \geq 1$ and $\delta>0$. There exists a constant $\bar{C}_{s}$ such that

$$
\|f g\|_{G_{\sigma, s-1}^{\delta}(\mathbb{R})} \leq \bar{C}_{s}\|f\|_{G_{\sigma, s-1}^{\delta}(\mathbb{R})}\|g\|_{G_{\sigma, s}^{\delta}(\mathbb{R})}
$$

Proof. By the similar argument as in Proposition 2.5, we have

$$
\|f g\|_{G_{\sigma, s-1}^{\delta}(\mathbb{R})}^{2} \leq\left\|\left(e^{\delta(-\Delta)^{\frac{1}{2 \sigma}}} f\right) \cdot\left(e^{\delta(-\Delta)^{\frac{1}{2 \sigma}}} g\right)\right\|_{H^{s-1}(\mathbb{R})}^{2} .
$$

Using the fact that $\|a b\|_{H^{s-1}(\mathbb{R})} \leq \bar{C}_{s}\|a\|_{H^{s-1}(\mathbb{R})}\|b\|_{H^{s}(\mathbb{R})}$ if $s>\frac{1}{2}$, we get

$\left\|\left(e^{\delta(-\Delta)^{\frac{1}{2 \sigma}}} f\right) \cdot\left(e^{\delta(-\Delta)^{\frac{1}{2 \sigma}}} g\right)\right\|_{H^{s-1}(\mathbb{R})}^{2} \leq \bar{C}_{s}\left\|e^{\delta(-\Delta)^{\frac{1}{2 \sigma}}} f\right\|_{H^{s-1}(\mathbb{R})}^{2}\left\|e^{\delta(-\Delta)^{\frac{1}{2 \sigma}}} g\right\|_{H^{s}(\mathbb{R})}^{2}=\bar{C}_{s}\|f\|_{G_{\sigma, s-1}^{\delta}(\mathbb{R})}^{2}\|g\|_{G_{\sigma, s}^{\delta}(\mathbb{R})}^{2}$.

Through out this paper, we use the notations $P_{1} \doteq\left(1-\partial_{x x}\right)^{-1}, P_{2} \doteq\left(4-\partial_{x x}\right)^{-1}, P_{3} \doteq \partial_{x}$ and $P_{i j} \doteq P_{i} P_{j}$ with $1 \leq i, j \leq 3$.

Proposition 2.7. If $s \in \mathbb{R}, \sigma, \delta>0$ and $f \in G_{\sigma, s}^{\delta}(\mathbb{R})$, then

$$
\left\|P_{1} f\right\|_{G_{\sigma, s}^{\delta}(\mathbb{R})}=\|f\|_{G_{\sigma, s-2}^{\delta}(\mathbb{R})} \leq\|f\|_{G_{\sigma, s}^{\delta}(\mathbb{R})}
$$




$$
\begin{aligned}
\left\|P_{2} f\right\|_{G_{\sigma, s}^{\delta}(\mathbb{R})} & \leq \frac{1}{4}\|f\|_{G_{\sigma, s}^{\delta}(\mathbb{R})}, \\
\left\|P_{13} f\right\|_{G_{\sigma, s}^{\delta}(\mathbb{R})} & \leq\|f\|_{G_{\sigma, s-1}^{\delta}(\mathbb{R})}, \\
\left\|P_{13} f\right\|_{G_{\sigma, s}^{\delta}(\mathbb{R})} & \leq \frac{1}{2}\|f\|_{G_{\sigma, s}^{\delta}(\mathbb{R})}, \\
\left\|P_{23} f\right\|_{G_{\sigma, s}^{\delta}(\mathbb{R})} & \leq \frac{1}{4}\|f\|_{G_{\sigma, s}^{\delta}(\mathbb{R})} .
\end{aligned}
$$

Proof. Since $\mathscr{F}\left[P_{1} f\right]=\frac{\widehat{f}(\xi)}{1+|\xi|^{2}}, \mathscr{F}\left[P_{2} f\right]=\frac{\widehat{f}(\xi)}{4+|\xi|^{2}}, \mathscr{F}\left[P_{13} f\right]=\frac{i \xi \widehat{f}(\xi)}{1+|\xi|^{2}}$ and $\mathscr{F}\left[P_{23} f\right]=\frac{i \xi \widehat{f}(\xi)}{4+|\xi|^{2}}$, it follows that

$$
\begin{aligned}
&\left\|P_{1} f\right\|_{G_{\sigma, s}^{\delta}(\mathbb{R})}=\left(\int_{\mathbb{R}}\left(1+|\xi|^{2}\right)^{s-2} e^{2 \delta|\xi|}|\widehat{f}(\xi)|^{2} d \xi\right)^{\frac{1}{2}}=\|f\|_{G_{\sigma, s-2}^{\delta}(\mathbb{R})} \leq\|f\|_{G_{\sigma, s}^{\delta}(\mathbb{R})}, \\
&\left\|P_{2} f\right\|_{G_{\sigma, s}^{\delta}(\mathbb{R})}=\left(\int_{\mathbb{R}} \frac{\left(1+|\xi|^{2}\right)^{s}}{\left(4+|\xi|^{2}\right)^{2}} e^{2 \delta|\xi|}|\widehat{f}(\xi)|^{2} d \xi\right)^{\frac{1}{2}} \leq \frac{1}{4}\left(\int_{\mathbb{R}}\left(1+|\xi|^{2}\right)^{s} e^{2 \delta|\xi|}|\widehat{f}(\xi)|^{2} d \xi\right)^{\frac{1}{2}}=\frac{1}{4}\|f\|_{G_{\sigma, s}^{\delta}(\mathbb{R}),} \\
&\left\|P_{13} f\right\|_{G_{\sigma, s}^{\delta}(\mathbb{R})}=\left(\int_{\mathbb{R}}\left(1+|\xi|^{2}\right)^{s-2}|\xi|^{2} e^{2 \delta|\xi|}|\widehat{f}(\xi)|^{2} d \xi\right)^{\frac{1}{2}} \leq\left(\int_{\mathbb{R}}\left(1+|\xi|^{2}\right)^{s-1} e^{2 \delta|\xi|}|\widehat{f}(\xi)|^{2} d \xi\right)^{\frac{1}{2}}=\|f\|_{G_{\sigma, s-1}^{\delta}(\mathbb{R})}, \\
&\left\|P_{13} f\right\|_{G_{\sigma, s}^{\delta}(\mathbb{R})}=\left(\int_{\mathbb{R}}\left(1+|\xi|^{2}\right)^{s-2}|\xi|^{2} e^{2 \delta|\xi|}|\widehat{f}(\xi)|^{2} d \xi\right)^{\frac{1}{2}} \leq\left(\int_{\mathbb{R}} \frac{\left(1+|\xi|^{2}\right)^{s}}{4} e^{2 \delta|\xi|}|\widehat{f}(\xi)|^{2} d \xi\right)^{\frac{1}{2}}=\frac{1}{2}\|f\|_{G_{\sigma, s}^{\delta}(\mathbb{R})}, \\
&\left\|P_{23} f\right\|_{G_{\sigma, s}^{\delta}(\mathbb{R})}=\left(\int_{\mathbb{R}} \frac{\left(1+|\xi|^{2}\right)^{s}|\xi|^{2}}{\left(4+|\xi|^{2}\right)^{2}} e^{2 \delta|\xi|}|\widehat{f}(\xi)|^{2} d \xi\right)^{\frac{1}{2}} \leq \frac{1}{4}\left(\int_{\mathbb{R}}\left(1+|\xi|^{2}\right)^{s} e^{2 \delta|\xi|}|\widehat{f}(\xi)|^{2} d \xi\right)^{\frac{1}{2}}=\frac{1}{4}\|f\|_{G_{\sigma, s}^{\delta}(\mathbb{R})} .
\end{aligned}
$$

Notations. Since all function spaces in the following sections are over $\mathbb{R}$, for simplicity, we drop $\mathbb{R}$ in the notation of function spaces if there is no ambiguity.

\section{A generalized Ovsyannikov theorem}

In order to study the Gevrey regularity of (1.1), we need the following generalized Ovsyannikov theorem.

Theorem 3.1. Let $\left\{X_{\delta}\right\}_{0<\delta<1}$ be a scale of decreasing Banach spaces, namely, for any $\delta^{\prime}<\delta$ we have $X_{\delta} \subset X_{\delta^{\prime}}$ and $\|\cdot\|_{\delta^{\prime}} \leq\|\cdot\|_{\delta}$. Consider the Cauchy problem

$$
\left\{\begin{array}{l}
\frac{d u}{d t}=F(t, u(t)), \\
\left.u\right|_{t=0}=u_{0} .
\end{array}\right.
$$

Let $T, R>0, \sigma \geq 1$. For given $u_{0} \in X_{1}$, assume that $F$ satisfies the following conditions:

(1) If for $0<\delta^{\prime}<\delta<1$ the function $t \mapsto u(t)$ is holomorphic in $|t|<T$ and continuous on $|t|<T$ with values in $X_{s}$ and

$$
\sup _{|t|<T}\|u(t)\|_{\delta}<R
$$


then $t \mapsto F(t, u(t))$ is a holomorphic function on $|t|<T$ with values in $X_{\delta^{\prime}}$.

(2) For any $0<\delta^{\prime}<\delta<1$ and any $u, v \in \overline{B\left(u_{0}, R\right)} \subset X_{\delta}$, there exists a positive constant $L$ depending on $u_{0}$ and $R$ such that

$$
\sup _{|t|<T}\|F(t, u)-F(t, v)\|_{\delta^{\prime}} \leq \frac{L}{\left(\delta-\delta^{\prime}\right)^{\sigma}}\|u-v\|_{\delta} .
$$

(3) For any $0<\delta<1$, there exists a positive constant $M$ depending on $u_{0}$ and $R$ such that

$$
\sup _{|t|<T}\left\|F\left(t, u_{0}\right)\right\|_{\delta} \leq \frac{M}{(1-\delta)^{\sigma}} .
$$

Then there exists a $T_{0} \in(0, T)$ and a unique solution $u(t)$ to the Cauchy problem (3.1), which for every $\delta \in(0,1)$ is holomorphic in $|t|<\frac{T_{0}(1-\delta)^{\sigma}}{2^{\sigma}-1}$ with values in $X_{\delta}$.

Remark 3.2. In fact, $T_{0}=\min \left\{\frac{1}{2^{2 \sigma+4} L}, \frac{\left(2^{\sigma}-1\right) R}{\left(2^{\sigma}-1\right) 2^{2 \sigma+3} L R+M}\right\}$, which gives a lower bound of the lifespan.

Remark 3.3. If $\sigma=1$, Theorem 3.1 reduced to the so called abstract Cauchy-Kovalevsky theorem. The original results were first proposed by Ovsyannikov in [38], [39] and [40]. Later, Nirenberg [36], Nishida [37], Treves [44], [45], and Baouendi and Goulaouic [1], [2] developed a lot of different versions of this theorem.

The proof of Theorem 3.1 is based on the fixed point argument in some suitable Banach space. Now we introduce a new Banach space.

Definition 3.4. Let $\sigma \geq 1$. For any $a>0$ we denote by $E_{a}$ the space of functions $u(t)$ which for every $0<\delta<1$ and $|t|<\frac{a(1-\delta)^{\sigma}}{2^{\sigma}-1}$, are holomorphic and continuous functions of $t$ with values in $X_{\delta}$ such that

$$
\|u\|_{E_{a}}=\sup _{|t|<\frac{a(1-\delta)^{\sigma}}{2^{\sigma}-1}, 0<\delta<1}\left(\|u(t)\|_{\delta}(1-\delta)^{\sigma} \sqrt{1-\frac{|t|}{a(1-\delta)^{\sigma}}}\right)<+\infty .
$$

Proposition 3.5. Let $\sigma \geq 1$. For any $a>0$, the function space $E_{a}$ is a Banach space equipped with the norm $\|\cdot\|_{E_{a}}$.

Proof. Suppose that $\left(u_{n}\right)_{n \geq 1}$ is a Cauchy sequence in $E_{a}$, that is

$$
\left\|u_{n}-u_{m}\right\|_{E_{a}} \rightarrow 0, \quad \text { as } \quad n, m \rightarrow \infty
$$

By virtue of the definition of $E_{a}$, we deduce that for any $0<\delta<1$,

$$
\sup _{|t|<\frac{a(1-\delta) \sigma}{2^{\sigma}-1}}\left\|u_{n}-u_{m}\right\|_{\delta} \rightarrow 0, \quad \text { as } \quad n, m \rightarrow \infty .
$$


Since $X_{\delta}$ is a Banach space, it follows that there exists a $u_{\delta} \in X_{\delta}$ such that

$$
\sup _{|t|<\frac{a(1-\delta)^{\sigma}}{2^{\sigma}-1}}\left\|u_{n}-u_{\delta}\right\|_{\delta} \rightarrow 0, \quad \text { as } \quad n \rightarrow \infty .
$$

Now we claim that $u_{\delta}$ is independent on $\delta$. Indeed, if $\delta_{1} \neq \delta_{2}$, with loss of generality suppose that $\delta_{1}<\delta_{2}$, and we obtain that,

$$
\left\|u_{n}-u_{\delta_{2}}\right\|_{\delta_{1}} \leq\left\|u_{n}-u_{\delta_{2}}\right\|_{\delta_{2}} \rightarrow 0, \quad \text { as } \quad n \rightarrow \infty
$$

which leads to $u_{\delta_{1}}=u_{\delta_{2}}$. Thus, for any $0<\delta<1$, we have $u=u_{\delta} \in X_{\delta}$. Since $\left(u_{n}\right)_{n \geq 1}$ is a Cauchy sequence in $E_{a}$, for any $\varepsilon>0$, there exists a $N_{1}=N_{1}(\varepsilon)$ such that if $n, m \geq N_{1},\left\|u_{n}-u_{m}\right\|_{E_{a}} \leq \frac{\varepsilon}{2}$. Note that $\left\|u_{n}-u\right\|_{\delta} \stackrel{n \rightarrow \infty}{\longrightarrow} 0$ for any $0<\delta<1$. For any $\varepsilon>0$, there exists a $N_{2}(\delta)$ such that if $n \geq N_{2}(\delta),\left\|u_{n}-u\right\|_{\delta} \leq \frac{\varepsilon}{2}$. Defining that $N=N(\delta, \varepsilon)=\max \left\{N_{1}, N_{2}(\delta)\right\}+1$ for any $\varepsilon>0$ and $0<\delta<1$, we deduce that for any $n \geq N_{1}$

$$
\begin{aligned}
\left\|u_{n}-u\right\|_{\delta}(1-\delta)^{\sigma} \sqrt{1-\frac{|t|}{a(1-\delta)^{\sigma}}} & \leq\left\|u_{n}-u_{N}\right\|_{E_{a}}+\left\|u_{N}-u\right\|_{\delta}(1-\delta)^{\sigma} \sqrt{1-\frac{|t|}{a(1-\delta)^{\sigma}}} \\
& \leq\left\|u_{n}-u_{N}\right\|_{E_{a}}+\left\|u_{N}-u\right\|_{\delta} \leq \frac{\varepsilon}{2}+\frac{\varepsilon}{2}=\varepsilon
\end{aligned}
$$

Since $N_{1}$ is independent on $\delta$, it follows from the above inequality that $\left\|u_{n}-u\right\|_{E_{a}} \stackrel{n \rightarrow \infty}{\longrightarrow} 0$.

The following lemmas are crucial to prove Theorem 3.1

Lemma 3.6. Let $\sigma \geq 1$. For every $0<\delta<1$ and $0 \leq t<\frac{a(1-\delta)^{\sigma}}{2^{\sigma}-1}$ we have

$$
1-\delta>\left(\frac{1}{2}\right)^{1+\frac{1}{\sigma}}\left\{\left[(1-\delta)^{\sigma}-\frac{t}{a}\right]^{\frac{1}{\sigma}}+\left[(1-\delta)^{\sigma}+\left(2^{\sigma+1}-1\right) \frac{t}{a}\right]^{\frac{1}{\sigma}}\right\}
$$

Proof. Since $t<\frac{a(1-\delta)^{\sigma}}{2^{\sigma}-1}$, it follows that

$$
2(1-\delta)^{\sigma}>(1-\delta)^{\sigma}+\left(2^{\sigma}-1\right) \frac{t}{a}=\frac{1}{2}\left[(1-\delta)^{\sigma}-\frac{t}{a}\right]+\frac{1}{2}\left[(1-\delta)^{\sigma}+\left(2^{\sigma+1}-1\right) \frac{t}{a}\right] .
$$

Using the fact that $(x+y)^{p} \leq 2^{p-1}\left(x^{p}+y^{p}\right)$ with $p=\sigma, x=\left(\frac{1}{2}\left[(1-\delta)^{\sigma}-\frac{t}{a}\right]\right)^{\frac{1}{\sigma}}>0$ and $y=$ $\left(\frac{1}{2}\left[(1-\delta)^{\sigma}+\left(2^{\sigma+1}-1\right) \frac{t}{a}\right]\right)^{\frac{1}{\sigma}}>0$, we deduce that

$$
\frac{1}{2}\left[(1-\delta)^{\sigma}-\frac{t}{a}\right]+\frac{1}{2}\left[(1-\delta)^{\sigma}+\left(2^{\sigma+1}-1\right) \frac{t}{a}\right]=x^{\sigma}+y^{\sigma} \geq \frac{(x+y)^{\sigma}}{2^{\sigma-1}} .
$$

Plugging (3.4) into (3.3) yields that

$$
1-\delta>\frac{x+y}{2}=\left(\frac{1}{2}\right)^{1+\frac{1}{\sigma}}\left\{\left[(1-\delta)^{\sigma}-\frac{t}{a}\right]^{\frac{1}{\sigma}}+\left[(1-\delta)^{\sigma}+\left(2^{\sigma+1}-1\right) \frac{t}{a}\right]^{\frac{1}{\sigma}}\right\} .
$$


Lemma 3.7. Let $\sigma \geq 1$. For every $a>0, u \in E_{a}, 0<\delta<1$ and $0 \leq t<\frac{a(1-\delta)^{\sigma}}{2^{\sigma}-1}$ we have

$$
\int_{0}^{t} \frac{\|u(\tau)\|_{\delta(\tau)}}{(\delta(\tau)-\delta)^{\sigma}} d \tau \leq \frac{a 2^{2 \sigma+3}\|u\|_{E_{a}}}{(1-\delta)^{\sigma}} \sqrt{\frac{a(1-\delta)^{\sigma}}{a(1-\delta)^{\sigma}-t}}
$$

where $\delta(\tau)=\frac{1}{2}(1+\delta)+\left(\frac{1}{2}\right)^{2+\frac{1}{\sigma}}\left\{\left[(1-\delta)^{\sigma}-\frac{t}{a}\right]^{\frac{1}{\sigma}}-\left[(1-\delta)^{\sigma}+\left(2^{\sigma+1}-1\right) \frac{t}{a}\right]^{\frac{1}{\sigma}}\right\} \in(\delta, 1)$.

Proof. By virtue of the definition of $E_{a}$, we obtain

$$
\int_{0}^{t} \frac{\|u(\tau)\|_{\delta(\tau)}}{(\delta(\tau)-\delta)^{\sigma}} d \tau \leq\|u\|_{E_{a}} \int_{0}^{t} \frac{1}{(\delta(\tau)-\delta)^{\sigma}(1-\delta(\tau))^{\sigma} \sqrt{1-\frac{\tau}{a(1-\delta(\tau))^{\sigma}}}} d \tau
$$

Taking advantage of Lemma 3.6, we have

$$
\begin{aligned}
\delta(\tau)-\delta & =\frac{1}{2}(1-\delta)+\left(\frac{1}{2}\right)^{2+\frac{1}{\sigma}}\left\{\left[(1-\delta)^{\sigma}-\frac{\tau}{a}\right]^{\frac{1}{\sigma}}-\left[(1-\delta)^{\sigma}+\left(2^{\sigma+1}-1\right) \frac{\tau}{a}\right]^{\frac{1}{\sigma}}\right\} \\
& \geq\left(\frac{1}{2}\right)^{1+\frac{1}{\sigma}}\left[(1-\delta)^{\sigma}-\frac{\tau}{a}\right]^{\frac{1}{\sigma}}
\end{aligned}
$$

and

$$
\begin{aligned}
1-\delta(\tau) & =\frac{1}{2}(1-\delta)-\left(\frac{1}{2}\right)^{2+\frac{1}{\sigma}}\left\{\left[(1-\delta)^{\sigma}-\frac{\tau}{a}\right]^{\frac{1}{\sigma}}-\left[(1-\delta)^{\sigma}+\left(2^{\sigma+1}-1\right) \frac{\tau}{a}\right]^{\frac{1}{\sigma}}\right\} \\
& \geq\left(\frac{1}{2}\right)^{1+\frac{1}{\sigma}}\left[(1-\delta)^{\sigma}+\left(2^{\sigma+1}-1\right) \frac{\tau}{a}\right]^{\frac{1}{\sigma}}
\end{aligned}
$$

which leads to

$$
(1-\delta(\tau))^{\sigma} \geq\left(\frac{1}{2}\right)^{\sigma+1}\left[(1-\delta)^{\sigma}-\frac{\tau}{a}\right]+\frac{\tau}{a}
$$

or equivalently

$$
a(1-\delta(\tau))^{\sigma}-\tau \geq\left(\frac{1}{2}\right)^{\sigma+1}\left[a(1-\delta)^{\sigma}-\tau\right] .
$$

Plugging (3.7)-(3.10) into (3.6) yields that

$$
\begin{aligned}
\int_{0}^{t} \frac{\|u(\tau)\|_{\delta(\tau)}}{(\delta(\tau)-\delta)^{\sigma}} d \tau & \leq\|u\|_{E_{a}} \int_{0}^{t} \frac{a^{2}}{\left[a(1-\delta)^{\sigma}-\tau\right]^{\frac{3}{2}}\left[a(1-\delta)^{\sigma}+\left(2^{\sigma+1}-1\right) \tau\right]^{\frac{1}{2}}} d \tau \\
& =\frac{a 2^{2(\sigma+1)}}{(1-\delta)^{\sigma}}\|u\|_{E_{a}} \int_{0}^{\frac{t}{a(1-\delta)^{\sigma}}} \frac{1}{(1-\theta)^{\frac{3}{2}}\left(1+\left(2^{\sigma+1}-1\right) \theta\right)^{\frac{1}{2}}} d \theta \\
& \leq \frac{a 2^{2(\sigma+1)}}{(1-\delta)^{\sigma}}\|u\|_{E_{a}} \int_{0}^{\frac{t}{a(1-\delta)^{\sigma}}} \frac{1}{(1-\theta)^{\frac{3}{2}}} d \theta \leq \frac{a 2^{2 \sigma+3}\|u\|_{E_{a}}}{(1-\delta)^{\sigma}} \sqrt{\frac{a(1-\delta)^{\sigma}}{a(1-\delta)^{\sigma}-t}}
\end{aligned}
$$

Proof of Theorem 3.1; We only consider the case $t \geq 0$. For any $t<\frac{a(1-\delta)^{\sigma}}{2^{\sigma}-1}$ with $a>0$ and $u(t) \in \overline{B\left(u_{0}, R\right)} \subset E_{a}$, we define that

$$
G(u(t)) \doteq u_{0}+\int_{0}^{t} F(\tau, u(\tau)) d \tau
$$


Since (3.1) is equivalent to

$$
u(t)=u_{0}+\int_{0}^{t} F(\tau, u(\tau)) d \tau,
$$

it follows that our initial value problem (3.1) can be reduced to find the fixed point of the operator $G$.

Step 1: If $u(t) \in E_{a}$, by virtue of Definition 3.4 we have $u(t)$ is a holomorphic and continuous function of $t$ with values in $X_{\delta}$ for any $0<\delta<1$. The condition (1) of $F$ implies that $F(t, u(t))$ is a holomorphic function of $t$ with values in $X_{\delta}$ for any $0<\delta<1$, which leads to $G(u(t))$ is a holomorphic and continuous function of $t$ with values in $X_{\delta}$ for any $0<\delta<1$. In addition, if $\left\|u-u_{0}\right\|_{E_{a}} \leq R$, we deduce from Lemma 3.7 and conditions (2)-(3) that

$$
\begin{aligned}
\left\|G(u(t))-u_{0}\right\|_{\delta} & \leq \int_{0}^{t}\|F(\tau, u(\tau))\|_{\delta} d \tau \leq \int_{0}^{t}\left\|F(\tau, u(\tau))-F\left(\tau, u_{0}\right)\right\|_{\delta} d \tau+\int_{0}^{t}\left\|F\left(\tau, u_{0}\right)\right\|_{\delta} d \tau \\
& \leq \int_{0}^{t} \frac{L\left\|u-u_{0}\right\|_{\delta(\tau)}}{(\delta(\tau)-\delta)^{\sigma}} d \tau+\frac{t M}{(1-\delta)^{\sigma}} \leq \frac{a 2^{2 \sigma+3} L R}{(1-\delta)^{\sigma}} \sqrt{\frac{a(1-\delta)^{\sigma}}{a(1-\delta)^{\sigma}-t}}+\frac{t M}{(1-\delta)^{\sigma}},
\end{aligned}
$$

which implies that

$$
\left\|G(u(t))-u_{0}\right\|_{E_{a}} \leq a 2^{2 \sigma+3} L R+\frac{a M}{2^{\sigma}-1} .
$$

By taking $a \leq \frac{\left(2^{\sigma}-1\right) R}{\left(2^{\sigma}-1\right) 2^{2 \sigma+3} L R+M}$, we verify that $G u \in \overline{B\left(u_{0}, R\right)} \subset E_{a}$, which leads to $G$ maps $\overline{B\left(u_{0}, R\right)} \subset E_{a}$ into itself.

Step 2: Assume that $u(t), v(t) \in \overline{B\left(u_{0}, R\right)} \subset E_{a}$. Taking advantage of Lemma 3.7 and the condition (2), we infer that

$$
\begin{aligned}
\|G(u(t))-G(v(t))\|_{\delta} & \leq \int_{0}^{t}\|F(\tau, u(\tau))-F(\tau, v(\tau))\|_{\delta} d \tau \leq \\
& \leq \int_{0}^{t} \frac{L\|u-v\|_{\delta(\tau)}}{(\delta(\tau)-\delta)^{\sigma}} d \tau \leq \frac{a 2^{2 \sigma+3} L\|u-v\|_{E_{a}}}{(1-\delta)^{\sigma}} \sqrt{\frac{a(1-\delta)^{\sigma}}{a(1-\delta)^{\sigma}-t}}
\end{aligned}
$$

which leads to

$$
\|G(u(t))-G(v(t))\|_{E_{a}} \leq a 2^{2 \sigma+3} L\|u-v\|_{E_{a}} .
$$

By taking $a \leq \frac{1}{2^{2 \sigma+4} L}$, we obtain $\|G(u(t))-G(v(t))\|_{E_{a}} \leq \frac{1}{2}\|u-v\|_{E_{a}}$, and hence $G$ is a contraction map on $\overline{B\left(u_{0}, R\right)} \subset E_{a}$. From Step 1 and Step 2, we deduce that if $a \leq T_{0}=\min \left\{\frac{1}{2^{2 \sigma+4} L}, \frac{\left(2^{\sigma}-1\right) R}{\left(2^{\sigma}-1\right) 2^{2 \sigma+3} L R+M}\right\}$, $T$ has a unique fixed point in $\overline{B\left(u_{0}, R\right)} \subset E_{a}$.

\section{Gevrey regularity and analyticity}

In this section we investigate the Gevrey regularity and analyticity of solutions to the Camassa-Holm type systems. By virtue of Remark 2.2, the case $\sigma>1$ is corresponding to Gevrey regularity while $\sigma=1$ is corresponding to analyticity. Our main results can be stated as follows. 
Theorem 4.1. Let $\sigma \geq 1$ and $s>\frac{3}{2}$. Assume that $u_{0} \in G_{\sigma, s}^{1}(\mathbb{R})$. Then for every $0<\delta<1$, there exists a $T_{0}>0$ such that the Camassa-Holm equation has a unique solution $u$ which is holomorphic in $|t|<\frac{T_{0}(1-\delta)^{\sigma}}{2^{\sigma}-1}$ with values in $G_{\sigma, s}^{\delta}(\mathbb{R})$. Moreover $T_{0} \approx \frac{1}{\left\|u_{0}\right\|_{G_{\sigma, s}^{1}(\mathbb{R})}}$.

Proof. In order to use Theorem 3.1, we rewrite $(\mathrm{CH})$ as follows:

$$
\left\{\begin{array}{l}
u_{t}=F(u) \doteq-u P_{3} u-P_{13}\left[u^{2}+\frac{1}{2}\left(P_{3} u\right)^{2}\right], \\
\left.u\right|_{t=0}=u_{0} .
\end{array}\right.
$$

For a fixed $\sigma \geq 1$ and $s>\frac{3}{2}$. By virtue of Proposition 2.3, we have $\left\{G_{\sigma, s}^{\delta}\right\}_{0<\delta<1}$ is a scale of decreasing Banach spaces. Let $C_{s}$ be the constant given in Proposition 2.5. By virtue of Propositions 2.4 2.5 and 2.7. we deduce that for any $0<\delta^{\prime}<\delta$,

$$
\begin{aligned}
\|F(u)\|_{G_{\sigma, s}^{\delta^{\prime}}} & \leq \frac{1}{2}\left\|P_{3}\left(u^{2}\right)\right\|_{G_{\sigma, s}^{\delta^{\prime}}}+\frac{1}{2}\left\|u^{2}\right\|_{G_{\sigma, s}^{\delta^{\prime}}}+\frac{1}{2}\left\|\left(P_{3} u\right)^{2}\right\|_{G_{\sigma, s-1}^{\delta^{\prime}}} \\
& \leq C_{s} \frac{e^{-\sigma} \sigma^{\sigma}}{2\left(\delta-\delta^{\prime}\right)^{\sigma}}\|u\|_{G_{\sigma, s}^{\delta}}^{2}+\frac{C_{s}}{2}\|u\|_{G_{\sigma, s}^{\delta}}^{2}+\frac{C_{s}}{2}\left\|P_{3} u\right\|_{G_{\sigma, s-1}^{\delta}}^{2} \leq \frac{C_{s}\left(e^{-\sigma} \sigma^{\sigma}+2\right)}{2\left(\delta-\delta^{\prime}\right)^{\sigma}}\|u\|_{G_{\sigma, s}^{\delta}}^{2},
\end{aligned}
$$

which implies that $F$ satisfies the condition (1) of Theorem 3.1. By the same token, we obtain that $\left\|F\left(u_{0}\right)\right\|_{G_{\sigma, s}^{\delta}} \leq \frac{C_{s}\left(e^{-\sigma} \sigma^{\sigma}+2\right)}{2(1-\delta)^{\sigma}}\left\|u_{0}\right\|_{G_{\sigma, s}^{1}}^{2}$. Thus, we see that $F$ satisfies the condition (3) of Theorem 3.1 with $M=C_{s}\left(\frac{e^{-\sigma} \sigma^{\sigma}}{2}+1\right)\left\|u_{0}\right\|_{G_{\sigma, s}^{1}}^{2}$. In order to prove our desire result, it suffices to show that $F$ satisfies the condition (2) of Theorem 3.1. Assume that $\left\|u-u_{0}\right\|_{G_{\sigma, s}^{\delta}} \leq R$ and $\left\|v-u_{0}\right\|_{G_{\sigma, s}^{\delta}} \leq R$. Applying Propositions 2.4 and 2.7 we get

$$
\begin{aligned}
\|F(u)-F(v)\|_{G_{\sigma, s}^{\delta^{\prime}}} & \leq \frac{e^{-\sigma} \sigma^{\sigma}}{2\left(\delta-\delta^{\prime}\right)^{\sigma}}\left\|u^{2}-v^{2}\right\|_{G_{\sigma, s}^{\delta}}+\left\|P_{13}\left(u^{2}-v^{2}\right)\right\|_{G_{\sigma, s}^{\delta^{\prime}}}+\frac{1}{2}\left\|P_{13}\left[\left(P_{3} u\right)^{2}-\left(P_{3} v\right)^{2}\right]\right\|_{G_{\sigma, s}^{\delta^{\prime}}} \\
& \leq \frac{e^{-\sigma} \sigma^{\sigma}}{2\left(\delta-\delta^{\prime}\right)^{\sigma}}\left\|u^{2}-v^{2}\right\|_{G_{\sigma, s}^{\delta}}+\frac{1}{2}\left\|u^{2}-v^{2}\right\|_{G_{\sigma, s}^{\delta}}+\frac{1}{2}\left\|\left(P_{3} u\right)^{2}-\left(P_{3} v\right)^{2}\right\|_{G_{\sigma, s-1}^{\delta}} \\
& \leq \frac{C_{s}\left(e^{-\sigma} \sigma^{\sigma}+1\right)}{2\left(\delta-\delta^{\prime}\right)^{\sigma}}\|u+v\|_{G_{\sigma, s}^{\delta}}\|u-v\|_{G_{\sigma, s}^{\delta}}+\frac{C_{s}}{2\left(\delta-\delta^{\prime}\right)^{\sigma}}\left\|P_{3} u+P_{3} v\right\|_{G_{\sigma, s-1}^{\delta}}\left\|P_{3} u-P_{3} v\right\|_{G_{\sigma, s-1}^{\delta}} \\
& \leq \frac{C_{s}\left(e^{-\sigma} \sigma^{\sigma}+1\right)}{2\left(\delta-\delta^{\prime}\right)^{\sigma}}\|u+v\|_{G_{\sigma, s}^{\delta}}\|u-v\|_{G_{\sigma, s}^{\delta}}+\frac{C_{s}}{2\left(\delta-\delta^{\prime}\right)^{\sigma}}\|u+v\|_{G_{\sigma, s}^{\delta}}\|u-v\|_{G_{\sigma, s}^{\delta}} \\
& \leq \frac{C_{s}\left(e^{-\sigma} \sigma^{\sigma}+2\right)}{2\left(\delta-\delta^{\prime}\right)^{\sigma}}\left(\|u\|_{G_{\sigma, s}^{\delta}}+\|v\|_{G_{\sigma, s}^{\delta}}\right)\|u-v\|_{G_{\sigma, s}^{\delta}} \\
& \leq \frac{C_{s}\left(e^{-\sigma} \sigma^{\sigma}+2\right)}{\left(\delta-\delta^{\prime}\right)^{\sigma}}\left(\left\|u_{0}\right\|_{G_{\sigma, s}^{\delta}}+R\right)\|u-v\|_{G_{\sigma, s}^{\delta}} \leq \frac{C_{s}\left(e^{-\sigma} \sigma^{\sigma}+2\right)}{\left(\delta-\delta^{\prime}\right)^{\sigma}}\left(\left\|u_{0}\right\|_{G_{\sigma, s}^{1}}+R\right)\|u-v\|_{G_{\sigma, s}^{\delta}} .
\end{aligned}
$$

From the above inequality, we verify that $F$ satisfies the condition (2) of Theorem 3.1 with $L=$ $C_{s}\left(e^{-\sigma} \sigma^{\sigma}+2\right)\left(\left\|u_{0}\right\|_{G_{\sigma, s}^{1}}+R\right)$. Moreover, $T_{0}=\min \left\{\frac{1}{2^{2 \sigma+4} L}, \frac{\left(2^{\sigma}-1\right) R}{\left(2^{\sigma}-1\right) 2^{2 \sigma+3} L R+M}\right\}$, by setting $R=\left\|u_{0}\right\|_{G_{\sigma, s}^{1}}$, we see that $L=2 C_{s}\left(e^{-\sigma} \sigma^{\sigma}+2\right)\left\|u_{0}\right\|_{G_{\sigma, s}^{1}}$ and $M \leq 2^{2 \sigma+3} L R$. Then, we have $T_{0}=\frac{1}{2^{2 \sigma+5} C_{s}\left(e^{-\sigma} \sigma^{\sigma}+2\right)\left\|u_{0}\right\|_{G_{\sigma, s}^{1}}}$. 
Theorem 4.2. Let $\sigma \geq 1$ and $s>\frac{3}{2}$. Assume that $u_{0} \in G_{\sigma, s}^{1}(\mathbb{R})$ and $\rho_{0} \in G_{\sigma, s-1}^{1}(\mathbb{R})$. Then for every $0<\delta<1$, there exists a $T_{0}>0$ such that the two-component Camassa-Holm system has a unique solution $(u, \rho)$ which is holomorphic in $|t|<\frac{T_{0}(1-\delta)^{\sigma}}{2^{\sigma}-1}$ with values in $G_{\sigma, s}^{\delta}(\mathbb{R}) \times G_{\sigma, s-1}^{\delta}(\mathbb{R})$. Moreover $T_{0} \approx \frac{1}{\left\|u_{0}\right\|_{G_{\sigma, s}^{1}(\mathbb{R})}+\left\|\rho_{0}\right\|_{G_{\sigma, s-1}^{1}}(\mathbb{R})}$.

Proof. We only consider the case $k=1$, and change the 2-component Camassa-Holm $(2 \mathrm{CH})$ system into the following form

$$
\left\{\begin{array}{l}
z_{t}=F(z) \\
\left.z\right|_{t=0}=z_{0}
\end{array}\right.
$$

where $z=(u, \rho)^{\mathrm{T}}, z_{0}=\left(u_{0}, \rho_{0}\right)^{\mathrm{T}}$ and

$$
F(z)=\left(\begin{array}{c}
F_{1}(z) \\
F_{2}(z)
\end{array}\right)=\left(\begin{array}{c}
-P_{3}\left(\frac{u^{2}}{2}\right)-P_{13}\left[u^{2}+\frac{1}{2}\left(P_{3} u\right)^{2}+\frac{1}{2} \rho^{2}\right] \\
-P_{3}(u \rho)
\end{array}\right) .
$$

For fixed $\sigma \geq 1$ and $s>\frac{3}{2}$, we set $X_{\delta}=G_{\sigma, s}^{\delta}(\mathbb{R}) \times G_{\sigma, s-1}^{\delta}(\mathbb{R})$ and

$$
\|z\|_{\delta}=\|u\|_{G_{\sigma, s}^{\delta}}+\|\rho\|_{G_{\sigma, s-1}^{\delta}}
$$

Thanks to Proposition 2.3, we have $\left\{X_{\delta}\right\}_{0<\delta<1}$ is a scale of decreasing Banach spaces. Let $C_{s}$ be the constant given in Proposition 2.5. According to Propositions 2.4, 2.5 and 2.7, we have for any $0<\delta^{\prime}<\delta$,

$$
\begin{aligned}
\left\|F_{1}(z)\right\|_{G_{\sigma, s}^{\delta^{\prime}}} & \leq \frac{1}{2}\left\|P_{3}\left(u^{2}\right)\right\|_{G_{\sigma, s}^{\delta^{\prime}}}+\frac{1}{2}\left\|u^{2}\right\|_{G_{\sigma, s}^{\delta^{\prime}}}+\frac{1}{2}\left\|\left(P_{3} u\right)^{2}\right\|_{G_{\sigma, s-1}^{\delta^{\prime}}}+\frac{1}{2}\left\|\rho^{2}\right\|_{G_{\sigma, s-1}^{\delta^{\prime}}} \\
& \leq C_{s} \frac{e^{-\sigma} \sigma^{\sigma}}{2\left(\delta-\delta^{\prime}\right)^{\sigma}}\|u\|_{G_{\sigma, s}^{\delta}}^{2}+\frac{C_{s}}{2}\|u\|_{G_{\sigma, s}^{\delta}}^{2}+\frac{C_{s}}{2}\left\|P_{3} u\right\|_{G_{\sigma, s-1}^{\delta}}^{2}+\frac{C_{s}}{2}\|\rho\|_{G_{\sigma, s-1}^{\delta}}^{2} \\
& \leq \frac{C_{s}\left(e^{-\sigma} \sigma^{\sigma}+2\right)}{2\left(\delta-\delta^{\prime}\right)^{\sigma}}\|u\|_{G_{\sigma, s}^{\delta}}^{2}+\frac{C_{s}}{2\left(\delta-\delta^{\prime}\right)^{\sigma}}\|\rho\|_{G_{\sigma, s-1}^{\delta}}^{2} \leq \frac{C_{s}\left(e^{-\sigma} \sigma^{\sigma}+3\right)}{2\left(\delta-\delta^{\prime}\right)^{\sigma}}\|z\|_{\delta}^{2}, \\
\left\|F_{2}(z)\right\|_{G_{\sigma, s-1}^{\delta^{\prime}}} & \leq \frac{e^{-\sigma} \sigma^{\sigma}}{\left(\delta-\delta^{\prime}\right)^{\sigma}}\|u \rho\|_{G_{\sigma, s-1}^{\delta}} \leq \frac{C_{s} e^{-\sigma} \sigma^{\sigma}}{\left(\delta-\delta^{\prime}\right)^{\sigma}}\|u\|_{G_{\sigma, s}^{\delta}}\|\rho\|_{G_{\sigma, s-1}^{\delta}} \leq \frac{C_{s} e^{-\sigma} \sigma^{\sigma}}{\left(\delta-\delta^{\prime}\right)^{\sigma}}\|z\|_{\delta}^{2},
\end{aligned}
$$

which imply that $\|F(z)\|_{\delta^{\prime}}=\left\|F_{1}(z)\right\|_{G_{\sigma, s}^{\delta^{\prime}}}+\left\|F_{2}(z)\right\|_{G_{\sigma, s-1}^{\delta^{\prime}}} \leq \frac{C_{s}\left(e^{-\sigma} \sigma^{\sigma}+5\right)}{2\left(\delta-\delta^{\prime}\right)^{\sigma}}\|z\|_{\delta}^{2}$ and $F$ satisfies the condition (1) of Theorem 3.1. By the same token, we obtain that $\left\|F\left(z_{0}\right)\right\|_{\delta} \leq \frac{C_{s}\left(e^{-\sigma} \sigma^{\sigma}+5\right)}{2(1-\delta)^{\sigma}}\left\|u_{0}\right\|_{G_{\sigma, s}^{1}}^{2}$. Thus, we see that $F$ satisfies the condition (3) of Theorem 3.1 with $M=\frac{C_{s}\left(e^{-\sigma} \sigma^{\sigma}+5\right)}{2}\left\|z_{0}\right\|_{1}^{2}$. In order to prove our desire result, it suffices to show that $F$ satisfies the condition (2) of Theorem 3.1 Assume that $\left\|z_{1}-z_{0}\right\|_{\delta} \leq R$ and $\left\|z_{2}-z_{0}\right\|_{\delta} \leq R$. Taking advantage of Propositions 2.4 and 2.7, we get

$\left\|F_{1}\left(z_{1}\right)-F_{1}\left(z_{2}\right)\right\|_{G_{\sigma, s}^{\delta^{\prime}}} \leq \frac{e^{-\sigma} \sigma^{\sigma}}{2\left(\delta-\delta^{\prime}\right)^{\sigma}}\left\|u_{1}^{2}-u_{2}^{2}\right\|_{G_{\sigma, s}^{\delta}}+\left\|P_{13}\left(u_{1}^{2}-u_{2}^{2}\right)\right\|_{G_{\sigma, s}^{\delta^{\prime}}}$ 


$$
\begin{aligned}
& +\frac{1}{2}\left\|P_{13}\left[\left(P_{3} u_{1}\right)^{2}-\left(P_{3} u_{2}\right)^{2}\right]\right\|_{G_{\sigma, s}^{\delta^{\prime}}}+\frac{1}{2}\left\|P_{13}\left(\rho_{1}^{2}-\rho_{2}^{2}\right)\right\|_{G_{\sigma, s}^{\delta^{\prime}}} \\
& \leq \frac{e^{-\sigma} \sigma^{\sigma}}{2\left(\delta-\delta^{\prime}\right)^{\sigma}}\left\|u_{1}^{2}-u_{2}^{2}\right\|_{G_{\sigma, s}^{\delta}}+\frac{1}{2}\left\|u_{1}^{2}-u_{2}^{2}\right\|_{G_{\sigma, s}^{\delta}} \\
& +\frac{1}{2}\left\|\left(P_{3} u_{1}\right)^{2}-\left(P_{3} u_{2}\right)^{2}\right\|_{G_{\sigma, s-1}^{\delta}}+\frac{1}{2}\left\|\rho_{1}^{2}-\rho_{2}^{2}\right\|_{G_{\sigma, s-1}^{\delta}} \\
& \leq \frac{C_{s}\left(e^{-\sigma} \sigma^{\sigma}+1\right)}{2\left(\delta-\delta^{\prime}\right)^{\sigma}}\left\|u_{1}+u_{2}\right\|_{G_{\sigma, s}^{\delta}}\left\|u_{1}-u_{2}\right\|_{G_{\sigma, s}^{\delta}} \\
& +\frac{C_{s}}{2\left(\delta-\delta^{\prime}\right)^{\sigma}}\left\|P_{3} u_{1}+P_{3} u_{2}\right\|_{G_{\sigma, s-1}^{\delta}}\left\|P_{3} u_{1}-P_{3} u_{2}\right\|_{G_{\sigma, s-1}^{\delta}}+\frac{C_{s}}{2}\left\|\rho_{1}+\rho_{2}\right\|_{G_{\sigma, s-1}^{\delta}}\left\|\rho_{1}-\rho_{2}\right\|_{G_{\sigma, s-1}^{\delta}} \\
& \leq \frac{C_{s}\left(e^{-\sigma} \sigma^{\sigma}+1\right)}{2\left(\delta-\delta^{\prime}\right)^{\sigma}}\left\|u_{1}+u_{2}\right\|_{G_{\sigma, s}^{\delta}}\left\|u_{1}-u_{2}\right\|_{G_{\sigma, s}^{\delta}}+\frac{C_{s}}{2\left(\delta-\delta^{\prime}\right)^{\sigma}}\left\|u_{1}+u_{2}\right\|_{G_{\sigma, s}^{\delta}}\left\|u_{1}-u_{2}\right\|_{G_{\sigma, s}^{\delta}} \\
& +\frac{C_{s}}{2}\left\|\rho_{1}+\rho_{2}\right\|\left\|_{G_{\sigma, s-1}^{\delta}}\right\| \rho_{1}-\rho_{2} \|_{G_{\sigma, s-1}^{\delta}} \\
& \leq \frac{C_{s}\left(e^{-\sigma} \sigma^{\sigma}+2\right)}{2\left(\delta-\delta^{\prime}\right)^{\sigma}}\left(\left\|z_{1}\right\|_{\delta}+\left\|z_{2}\right\| \|_{\delta}\right)\left\|z_{1}-z_{2}\right\|_{\delta} \\
& \leq \frac{C_{s}\left(e^{-\sigma} \sigma^{\sigma}+2\right)}{\left(\delta-\delta^{\prime}\right)^{\sigma}}\left(\left\|z_{0}\right\|_{\delta}+R\right)\left\|z_{1}-z_{2}\right\|_{\delta} \leq \frac{C_{s}\left(e^{-\sigma} \sigma^{\sigma}+2\right)}{\left(\delta-\delta^{\prime}\right)^{\sigma}}\left(\left\|z_{0}\right\|_{1}+R\right)\left\|z_{1}-z_{2}\right\|_{\delta},
\end{aligned}
$$

$$
\begin{aligned}
\left\|F_{2}\left(z_{1}\right)-F_{2}\left(z_{2}\right)\right\|_{G_{\sigma, s-1}^{\delta^{\prime}}} & \leq \frac{e^{-\sigma} \sigma^{\sigma}}{\left(\delta-\delta^{\prime}\right)^{\sigma}}\left\|u_{1} \rho_{1}-u_{2} \rho_{2}\right\|_{G_{\sigma, s-1}^{\delta}} \leq \frac{e^{-\sigma} \sigma^{\sigma}}{\left(\delta-\delta^{\prime}\right)^{\sigma}}\left[\left\|\left(u_{1}-u_{2}\right) \rho_{1}\right\|_{G_{\sigma, s-1}^{\delta}}+\left\|\left(\rho_{1}-\rho_{2}\right) u_{2}\right\|_{G_{\sigma, s-1}^{\delta}}\right] \\
& \leq \frac{C_{s} e^{-\sigma} \sigma^{\sigma}}{\left(\delta-\delta^{\prime}\right)^{\sigma}}\left(\left\|u_{1}-u_{2}\right\|_{G_{\sigma, s}^{\delta}}\left\|\rho_{1}\right\|_{G_{\sigma, s-1}^{\delta}}+\left\|u_{2}\right\|_{G_{\sigma, s}^{\delta}}\left\|\rho_{1}-\rho_{2}\right\|_{G_{\sigma, s-1}^{\delta}}\right) \\
& \leq \frac{C_{s} e^{-\sigma} \sigma^{\sigma}}{\left(\delta-\delta^{\prime}\right)^{\sigma}}\left(\left\|z_{1}\right\| \delta\left\|u_{1}-u_{2}\right\|_{G_{\sigma, s}^{\delta}}+\left\|z_{2}\right\|_{\delta}\left\|\rho_{1}-\rho_{2}\right\|_{G_{\sigma, s-1}^{\delta}}\right) \\
& \leq \frac{C_{s} e^{-\sigma} \sigma^{\sigma}}{\left(\delta-\delta^{\prime}\right)^{\sigma}}\left(\left\|z_{0}\right\|_{\delta}+R\right)\left(\left\|u_{1}-u_{2}\right\|_{G_{\sigma, s}^{\delta}}+\left\|\rho_{1}-\rho_{2}\right\|_{G_{\sigma, s-1}^{\delta}}\right) \\
& \leq \frac{C_{s} e^{-\sigma} \sigma^{\sigma}}{\left(\delta-\delta^{\prime}\right)^{\sigma}}\left(\left\|z_{0}\right\|_{1}+R\right)\left\|z_{1}-z_{2}\right\|_{\delta} .
\end{aligned}
$$

From the above inequalities, we verify that $\left\|F\left(z_{1}\right)-F\left(z_{2}\right)\right\|_{\delta^{\prime}}=\left\|F_{1}\left(z_{1}\right)-F_{1}\left(z_{2}\right)\right\|_{G_{\sigma, s}^{\delta^{\prime}}}+\| F_{2}\left(z_{1}\right)-$ $F_{2}\left(z_{2}\right)\left\|_{G_{\sigma, s-1}^{\delta^{\prime}}} \leq \frac{2 C_{s}\left(e^{-\sigma} \sigma^{\sigma}+1\right)}{\left(\delta-\delta^{\prime}\right)^{\sigma}}\left(\left\|z_{0}\right\|_{1}+R\right)\right\| z_{1}-z_{2} \|_{\delta}$ and $F$ satisfies the condition (2) of Theorem 3.1 with $L=2 C_{s}\left(e^{-\sigma} \sigma^{\sigma}+1\right)\left(\left\|z_{0}\right\|_{1}+R\right)$. Moreover, $T_{0}=\min \left\{\frac{1}{2^{2 \sigma+4} L}, \frac{\left(2^{\sigma}-1\right) R}{\left(2^{\sigma}-1\right) 2^{2 \sigma+3} L R+M}\right\}$, by setting $R=\left\|z_{0}\right\|_{1}$, we see that $L=4 C_{s}\left(e^{-\sigma} \sigma^{\sigma}+1\right)\left\|z_{0}\right\|_{1}$ and $M \leq 2^{2 \sigma+3} L R$. Then, we get that $T_{0}=$ $\frac{1}{2^{2 \sigma+6} C_{s}\left(e^{-\sigma} \sigma^{\sigma}+1\right)\left\|z_{0}\right\|_{1}}$.

Remark 4.3. By the similar argument as in the proof of the above theorem, one can obtain the Gevrey regularity and analyticity for the modify 2-component Camassa-Holm system (M2CH).

Theorem 4.4. Let $\sigma \geq 1$ and $s>\frac{1}{2}$. Assume that $\left(u_{0}, v_{0}, w_{0}\right) \in\left(G_{\sigma, s}^{1}(\mathbb{R})\right)^{3}$. Then for every $0<\delta<1$, there exists a $T_{0}>0$ such that the three-component Camassa-Holm system has a unique solution $(u, v, w)$ which is holomorphic in $|t|<\frac{T_{0}(1-\delta)^{\sigma}}{2^{\sigma}-1}$ with values in $\left(G_{\sigma, s}^{\delta}(\mathbb{R})\right)^{3}$. Moreover $T_{0} \approx$ 
$\frac{1}{\left(\left\|u_{0}\right\|_{G_{\sigma, s}^{1}}^{1}+\left\|v_{0}\right\|_{G_{\sigma, s}^{1}}^{1}+\left\|w_{0}\right\|_{G_{\sigma, s}^{1}}{ }^{2}+\left\|u_{0}\right\|_{G_{\sigma, s}^{1}}^{1}+\left\|v_{0}\right\|_{G_{\sigma, s}^{1}}+\left\|w_{0}\right\|_{G_{\sigma, s}^{1}}\right.}$.

Proof. By virtue of $(3 \mathrm{CH})$, we see that $a=P_{1} u, c=P_{1} w$ and $b=P_{2}\left(w \cdot P_{13} u-u \cdot P_{13} w\right)+2 P_{2}\left(P_{13} u\right.$.

$\left.P_{1} w-P_{1} u \cdot P_{13} w\right)-2 P_{2} v=B(u, w)-2 P_{2} v$. Hence, we change $(3 \mathrm{CH})$ into

$$
\left\{\begin{array}{c}
U_{t}=F(U), \\
\left.U\right|_{t=0}=U_{0},
\end{array}\right.
$$

where $U=(u, v, w)^{\mathrm{T}}, U_{0}=\left(u_{0}, v_{0}, w_{0}\right)^{\mathrm{T}}$ and

$$
\begin{aligned}
& \text { (4.11) } \quad F(U)=\left(\begin{array}{c}
F_{1}(U) \\
F_{2}(U) \\
F_{3}(U)
\end{array}\right) \\
& =\left(\begin{array}{c}
-v \cdot P_{13} u+P_{3} u\left(B(u, w)-2 P_{2} v\right)+\frac{3}{2} u\left(P_{3} B(u, w)-2 P_{23} v\right)-\frac{3}{2} u\left(P_{13} u \cdot P_{13} w-P_{1} u \cdot P_{1} w\right) \\
2 v \cdot P_{3} B(u, w)-4 v P_{23} v+P_{3} v \cdot B(u, w)-2 P_{3} v P_{2} v \\
-v \cdot P_{13} w+P_{3} w\left(B(u, w)-2 P_{2} v\right)+\frac{3}{2} w\left(P_{3} B(u, w)-2 P_{23} v\right)+\frac{3}{2} w\left(P_{13} u \cdot P_{13} w-P_{1} u \cdot P_{1} w\right)
\end{array}\right) .
\end{aligned}
$$

For fixed $\sigma \geq 1$ and $s>\frac{1}{2}$, we set $X_{\delta}=\left(G_{\sigma, s}^{\delta}(\mathbb{R})\right)^{3}$ and

$$
\|U\|_{\delta}=\|u\|_{G_{\sigma, s}^{\delta}}+\|v\|_{G_{\sigma, s}^{\delta}}+\|w\|_{G_{\sigma, s}^{\delta}} .
$$

Due to Proposition 2.3 we have $\left\{X_{\delta}\right\}_{0<\delta<1}$ is a scale of decreasing Banach spaces.

Let $C_{s}$ be the constant given in Proposition 2.5. Taking advantage of Propositions 2.4, 2.5 and 2.7. we verify that for any $0<\delta^{\prime}<\delta$

$$
\begin{aligned}
\left\|F_{1}(U)\right\|_{G_{\sigma, s}^{\delta^{\prime}}} \leq \frac{C_{s}}{2}\|v\|_{G_{\sigma, s}^{\delta^{\prime}}}\|u\|_{G_{\sigma, s}^{\delta^{\prime}}}+\frac{C_{s} e^{-\sigma} \sigma^{\sigma}}{\left(\delta-\delta^{\prime}\right)^{\sigma}}\|u\|_{G_{\sigma, s}^{\delta}}\left(\|B(u, w)\|_{G_{\sigma, s}^{\delta^{\prime}}}+\frac{1}{2}\|v\|_{G_{\sigma, s}^{\delta^{\prime}}}\right) \\
+\frac{3}{2} C_{s}\|u\|_{G_{\sigma, s}^{\delta^{\prime}}}\left(\left\|P_{3} B(u, w)\right\|_{G_{\sigma, s}^{\delta^{\prime}}}+\frac{1}{2}\|v\|_{G_{\sigma, s}^{\delta^{\prime}}}\right)+\frac{15}{8} C_{s}^{2}\|u\|_{G_{\sigma, s}^{\delta^{\prime}}}^{2}\|w\|_{G_{\sigma, s}^{\delta^{\prime}}} .
\end{aligned}
$$

Since $B(u, w)=P_{2}\left(w \cdot P_{13} u-u \cdot P_{13} w\right)+2 P_{2}\left(P_{13} u \cdot P_{1} w-P_{1} u \cdot P_{13} w\right)$, it follows that

$$
\|B(u, w)\|_{G_{\sigma, s}^{\delta^{\prime}}},\left\|P_{3} B(u, w)\right\|_{G_{\sigma, s}^{\delta^{\prime}}} \leq \frac{3}{4} C_{s}\|u\|_{G_{\sigma, s}^{\delta^{\prime}}}\|w\|_{G_{\sigma, s}^{\delta^{\prime}}} .
$$

Plugging (4.7) into (4.6) yields that

$$
\begin{aligned}
\left\|F_{1}(U)\right\|_{G_{\sigma, s}^{\delta^{\prime}}} & \leq \frac{5}{4} C_{s}\|u\|_{G_{\sigma, s}^{\delta^{\prime}}}\|v\|_{G_{\sigma, s}^{\delta^{\prime}}}+3 C_{s}^{2}\|u\|_{G_{\sigma, s}^{\delta^{\prime}}}^{2}\|w\|_{G_{\sigma, s}^{\delta^{\prime}}}+\frac{C_{s} e^{-\sigma} \sigma^{\sigma}}{\left(\delta-\delta^{\prime}\right)}\|u\|_{G_{\sigma, s}^{\delta}}\left(\frac{3}{4} C_{s}\|u\|_{G_{\sigma, s}^{\delta^{\prime}}}\|w\|_{G_{\sigma, s}^{\delta^{\prime}}}+\frac{1}{2}\|v\|_{G_{\sigma, s}^{\delta^{\prime}}}\right) \\
& \left.\leq \frac{C_{s}^{2}}{\left(\delta-\delta^{\prime}\right)^{\sigma}}\left(3+\frac{3 e^{-\sigma} \sigma^{\sigma}}{4}\right)\|u\|_{G_{\sigma, s}^{\delta}}^{2}\|w\|_{G_{\sigma, s}^{\delta}}+\frac{C_{s}}{\left(\delta-\delta^{\prime}\right)^{\sigma}}\left(\frac{7}{4}+\frac{e^{-\sigma} \sigma^{\sigma}}{2}\right)\|u\|_{G_{\sigma, s}^{\delta}}\|v\|_{G_{\sigma, s}^{\delta}}\right) \\
& \leq \frac{C_{s}\|U\|_{\delta}^{2}}{\left(\delta-\delta^{\prime}\right)^{\sigma}}\left[C_{s}\|U\|_{\delta}\left(3+\frac{3 e^{-\sigma} \sigma^{\sigma}}{4}\right)+\left(\frac{5}{4}+\frac{e^{-\sigma} \sigma^{\sigma}}{2}\right)\right] .
\end{aligned}
$$


By the same token, we have

$$
\begin{aligned}
&\left\|F_{2}(U)\right\|_{G_{\sigma, s}^{\delta^{\prime}}} \leq \frac{C_{s}\|U\|_{\delta}^{2}}{\left(\delta-\delta^{\prime}\right)^{\sigma}}\left[C_{s}\|U\|_{\delta}\left(\frac{3}{2}+\frac{3 e^{-\sigma} \sigma^{\sigma}}{4}\right)+\left(1+\frac{e^{-\sigma} \sigma^{\sigma}}{2}\right)\right], \\
&\left\|F_{3}(U)\right\|_{G_{\sigma, s}^{\delta^{\prime}}} \leq \frac{C_{s}\|U\|_{\delta}^{2}}{\left(\delta-\delta^{\prime}\right)^{\sigma}}\left[C_{s}\|U\|_{\delta}\left(3+\frac{3 e^{-\sigma} \sigma^{\sigma}}{4}\right)+\left(\frac{5}{4}+\frac{e^{-\sigma} \sigma^{\sigma}}{2}\right)\right],
\end{aligned}
$$

which lead to

$$
\|F(U)\|_{\delta^{\prime}}=\left\|F_{1}(U)\right\|_{G_{\sigma, s}^{\delta^{\prime}}}+\left\|F_{2}(U)\right\|_{G_{\sigma, s}^{\delta^{\prime}}}+\left\|F_{3}(U)\right\|_{G_{\sigma, s}^{\delta^{\prime}}} \leq \frac{C_{s}\|U\|_{\delta}^{2}}{\left(\delta-\delta^{\prime}\right)^{\sigma}}\left[C_{s}\|U\|_{\delta}\left(\frac{15}{2}+\frac{9 e^{-\sigma} \sigma^{\sigma}}{4}\right)+\left(\frac{9}{2}+\frac{3 e^{-\sigma} \sigma^{\sigma}}{2}\right)\right] .
$$

Thus, we verify that $F$ satisfies the condition (1) of Theorem 3.1 By the similar estimates as above, we obtain that

$$
\left\|F\left(U_{0}\right)\right\|_{\delta} \leq \frac{C_{s}\left\|U_{0}\right\|_{1}^{2}}{(1-\delta)^{\sigma}}\left[C_{s}\left\|U_{0}\right\|_{1}\left(\frac{15}{2}+\frac{9 e^{-\sigma} \sigma^{\sigma}}{4}\right)+\left(\frac{9}{2}+\frac{3 e^{-\sigma} \sigma^{\sigma}}{2}\right)\right]
$$

which implies that $F$ satisfies the condition (3) of Theorem 3.1 and $M=C_{s}\left\|U_{0}\right\|_{1}^{2}\left[C_{s}\left\|U_{0}\right\|_{1}\left(\frac{15}{2}+\right.\right.$ $\left.\left.\frac{9 e^{-\sigma} \sigma^{\sigma}}{4}\right)+\left(\frac{9}{2}+\frac{3 e^{-\sigma} \sigma^{\sigma}}{2}\right)\right]$. In order to prove our desire result, it suffices to show that $F$ satisfies the condition (2) of Theorem 3.1 Assume that $\left\|U_{1}-U_{0}\right\|_{\delta} \leq R$ and $\left\|U_{2}-U_{0}\right\|_{\delta} \leq R$ for every $0<\delta<1$. Making use of Propositions 2.5 and 2.7, we obtain that for any $0<\delta^{\prime}<\delta<1$

$$
\begin{aligned}
\|I\|_{G_{\sigma, s}^{\delta^{\prime}}} \doteq\left\|v_{1} P_{13} u_{1}-v_{2} P_{13} u_{2}\right\|_{G_{\sigma, s}^{\delta^{\prime}}} & \leq \frac{1}{2} C_{s}\left\|v_{1}-v_{2}\right\|_{G_{\sigma, s}^{\delta^{\prime}}}\left\|u_{1}\right\|_{G_{\sigma, s}^{\delta^{\prime}}}+\frac{1}{2} C_{s}\left\|u_{1}-u_{2}\right\|_{G_{\sigma, s}^{\delta^{\prime}}}\left\|v_{2}\right\|_{G_{\sigma, s}^{\delta^{\prime}}} \\
& \leq C_{s}\left\|U_{1}-U_{2}\right\|_{\delta}\left(\left\|U_{0}\right\|_{1}+R\right) \leq \frac{C_{s}\left(\left\|U_{0}\right\|_{1}+R\right)}{\left(\delta-\delta^{\prime}\right)^{\sigma}}\left\|U_{1}-U_{2}\right\|_{\delta},
\end{aligned}
$$

$$
\begin{aligned}
\|I I\|_{G_{\sigma, s}^{\delta^{\prime}}} \doteq\left\|P_{3} u_{1} P_{2} v_{1}-P_{3} u_{2} P_{2} v_{2}\right\|_{G_{\sigma, s}^{\delta^{\prime}}} & \leq \frac{C_{s} e^{-\sigma} \sigma^{\sigma}}{4\left(\delta-\delta^{\prime}\right)^{\sigma}}\left\|u_{1}-u_{2}\right\|_{G_{\sigma, s}^{\delta}}\left\|v_{1}\right\|_{G_{\sigma, s}^{\delta}}+\frac{C_{s} e^{-\sigma} \sigma^{\sigma}}{4\left(\delta-\delta^{\prime}\right)^{\sigma}}\left\|u_{2}\right\|_{G_{\sigma, s}^{\delta}}\left\|v_{1}-v_{2}\right\|_{G_{\sigma, s}^{\delta}} \\
& \leq \frac{C_{s}\left(\left\|U_{0}\right\|_{1}+R\right) e^{-\sigma} \sigma^{\sigma}}{2\left(\delta-\delta^{\prime}\right)^{\sigma}}\left\|U_{1}-U_{2}\right\|_{\delta},
\end{aligned}
$$

$$
\begin{aligned}
\|I I I\|_{G_{\sigma, s}^{\delta^{\prime}}} \doteq\left\|u_{1} P_{23} v_{1}-u_{2} P_{23} v_{2}\right\|_{G_{\sigma, s}^{\delta^{\prime}}} & \leq \frac{1}{4} C_{s}\left\|v_{1}-v_{2}\right\|_{G_{\sigma, s}^{\delta^{\prime}}}\left\|u_{1}\right\|_{G_{\sigma, s}^{\delta^{\prime}}}+\frac{1}{4} C_{s}\left\|u_{1}-u_{2}\right\|_{G_{\sigma, s}^{\delta^{\prime}}}\left\|v_{2}\right\|_{G_{\sigma, s}^{\delta^{\prime}}} \\
& \leq \frac{C_{s}}{2}\left\|U_{1}-U_{2}\right\|_{\delta}\left(\left\|U_{0}\right\|_{1}+R\right) \leq \frac{C_{s}\left(\left\|U_{0}\right\|_{1}+R\right)}{2\left(\delta-\delta^{\prime}\right)^{\sigma}}\left\|U_{1}-U_{2}\right\|_{\delta},
\end{aligned}
$$

$$
\|I V\|_{G_{\sigma, s}^{\delta^{\prime}}} \doteq\left\|u_{1} P_{13} u_{1} P_{13} w_{1}-u_{2} P_{13} u_{2} P_{13} w_{2}\right\|_{G_{\sigma, s}^{\delta^{\prime}}} \leq \frac{C_{s}^{2}}{4}\left\|u_{1}-u_{2}\right\|_{G_{\sigma, s}^{\delta^{\prime}}}\left\|u_{1}\right\|_{G_{\sigma, s}^{\delta^{\prime}}}\left\|w_{1}\right\|_{G_{\sigma, s}^{\delta^{\prime}}}
$$




$$
\begin{gathered}
+\frac{C_{s}^{2}}{4}\left\|u_{1}-u_{2}\right\|_{G_{\sigma, s}^{\delta^{\prime}}}\left\|u_{2}\right\|_{G_{\sigma, s}^{\delta^{\prime}}}\left\|w_{1}\right\|_{G_{\sigma, s}^{\delta^{\prime}}}+\frac{C_{s}^{2}}{4}\left\|w_{1}-w_{2}\right\|_{G_{\sigma, s}^{\delta^{\prime}}}\left\|u_{2}\right\|_{G_{\sigma, s}^{\delta^{\prime}}}^{2} \leq \frac{3 C_{s}^{2}\left(\left\|U_{0}\right\|_{1}+R\right)^{2}}{4\left(\delta-\delta^{\prime}\right)^{\sigma}}\left\|U_{1}-U_{2}\right\|_{\delta}, \\
\|V\|_{G_{\sigma, s}^{\delta^{\prime}}} \doteq\left\|u_{1} P_{1} u_{1} P_{1} w_{1}-u_{2} P_{1} u_{2} P_{1} w_{2}\right\|_{G_{\sigma, s}^{\delta^{\prime}}} \leq \frac{3 C_{s}^{2}\left(\left\|U_{0}\right\|_{1}+R\right)^{2}}{\left(\delta-\delta^{\prime}\right)^{\sigma}}\left\|U_{1}-U_{2}\right\|_{\delta},
\end{gathered}
$$

$\left\|B\left(u_{1}, w_{1}\right)-B\left(u_{2}, w_{2}\right)\right\|_{G_{\sigma, s}^{\delta^{\prime}}} \leq C\left(P_{2}\right)\left\|w_{1} P_{13} u_{1}-w_{2} P_{13} u_{2}\right\|_{G_{\sigma, s}^{\delta^{\prime}}}+C\left(P_{2}\right)\left\|u_{1} P_{13} w_{1}-u_{2} P_{13} w_{2}\right\|_{G_{\sigma, s}^{\delta^{\prime}}}$

$$
\begin{aligned}
& +2 C\left(P_{2}\right)\left\|P_{13} u_{1} P_{1} w_{1}-P_{13} u_{2} P_{1} w_{2}\right\|_{G_{\sigma, s}^{\delta^{\prime}}}+2 C\left(P_{2}\right)\left\|P_{13} w_{1} P_{1} u_{1}-P_{13} w_{2} P_{1} u_{2}\right\|_{G_{\sigma, s}^{\delta^{\prime}}} \\
& \leq \frac{3 C_{s}\left(\left\|U_{0}\right\|_{1}+R\right)}{2\left(\delta-\delta^{\prime}\right)^{\sigma}}\left\|U_{1}-U_{2}\right\|_{\delta},
\end{aligned}
$$

$\left\|P_{3} B\left(u_{1}, w_{1}\right)-P_{3} B\left(u_{2}, w_{2}\right)\right\| \leq C\left(P_{23}\right)\left\|w_{1} P_{13} u_{1}-w_{2} P_{13} u_{2}\right\|_{G_{\sigma, s}^{\delta^{\prime}}}+C\left(P_{23}\right)\left\|u_{1} P_{13} w_{1}-u_{2} P_{13} w_{2}\right\|_{G_{\sigma, s}^{\delta^{\prime}}}$

$$
\begin{aligned}
& +2 C\left(P_{23}\right)\left\|P_{13} u_{1} P_{1} w_{1}-P_{13} u_{2} P_{1} w_{2}\right\|_{G_{\sigma, s}^{\delta^{\prime}}}+2 C\left(P_{23}\right)\left\|P_{13} w_{1} P_{1} u_{1}-P_{13} w_{2} P_{1} u_{2}\right\|_{G_{\sigma, s}^{\delta^{\prime}}} \\
& \leq \frac{3 C_{s}\left(\left\|U_{0}\right\|_{1}+R\right)}{2\left(\delta-\delta^{\prime}\right)^{\sigma}}\left\|U_{1}-U_{2}\right\|_{\delta},
\end{aligned}
$$

$$
\begin{aligned}
\|V I\|_{G_{\sigma, s}^{\delta^{\prime}}} & \doteq\left\|P_{3} u_{1} B\left(u_{1}, w_{1}\right)-P_{3} u_{2} B\left(u_{2}, w_{2}\right)\right\|_{G_{\sigma, s}^{\delta^{\prime}}} \leq \frac{C_{s} e^{-\sigma} \sigma^{\sigma}}{\left(\delta-\delta^{\prime}\right)^{\sigma}}\left\|u_{1}-u_{2}\right\|_{G_{\sigma, s}^{\delta}}\left\|B\left(u_{1}, w_{1}\right)\right\|_{G_{\sigma, s}^{\delta^{\prime}}} \\
& +\frac{C_{s} e^{-\sigma} \sigma^{\sigma}}{\left(\delta-\delta^{\prime}\right)^{\sigma}}\left\|u_{2}\right\|_{G_{\sigma, s}^{\delta}}\left\|B\left(u_{1}, w_{1}\right)-B\left(u_{2}, w_{2}\right)\right\|_{G_{\sigma, s}^{\delta^{\prime}}} \leq \frac{9 C_{s}^{2} e^{-\sigma} \sigma^{\sigma}\left(\left\|U_{0}\right\|_{1}+R\right)^{2}}{4\left(\delta-\delta^{\prime}\right)^{\sigma}}\left\|U_{1}-U_{2}\right\|_{\delta},
\end{aligned}
$$

$$
\begin{aligned}
\|V I I\|_{G_{\sigma, s}^{\delta^{\prime}}} & \doteq\left\|u_{1} P_{3} B\left(u_{1}, w_{1}\right)-u_{2} P_{3} B\left(u_{2}, w_{2}\right)\right\|_{G_{\sigma, s}^{\delta^{\prime}}} \leq C_{s}\left\|u_{1}-u_{2}\right\|_{G_{\sigma, s}^{\delta^{\prime}}}\left\|P_{3} B\left(u_{1}, w_{1}\right)\right\|_{G_{\sigma, s}^{\delta^{\prime}}} \\
& +C_{s}\left\|u_{2}\right\|_{G_{\sigma, s}^{\delta^{\prime}}}\left\|P_{3} B\left(u_{1}, w_{1}\right)-P_{3} B\left(u_{2}, w_{2}\right)\right\|_{G_{\sigma, s}^{\delta^{\prime}}} \leq \frac{9 C_{s}^{2}\left(\left\|U_{0}\right\|_{1}+R\right)^{2}}{4\left(\delta-\delta^{\prime}\right)^{\sigma}}\left\|U_{1}-U_{2}\right\|_{\delta}
\end{aligned}
$$

Since $F_{1}\left(U_{1}\right)-F_{1}\left(U_{2}\right)=-I-2 I I-3 I I I-\frac{3}{2} I V+\frac{3}{2} V+V I+\frac{3}{2} V I I$, it follows from the above inequalities that

$$
\left\|F_{1}\left(U_{1}\right)-F_{1}\left(U_{2}\right)\right\|_{G_{\sigma, s}^{\delta^{\prime}}} \leq \frac{C_{s}\left\|U_{1}-U_{2}\right\|_{\delta}\left(\left\|U_{0}\right\|_{1}+R\right)}{\left(\delta-\delta^{\prime}\right)^{\sigma}}\left[C_{s}\left(\left\|U_{0}\right\|_{1}+R\right)\left(9+\frac{9 e^{-\sigma} \sigma^{\sigma}}{4}\right)+\left(\frac{5}{2}+e^{-\sigma} \sigma^{\sigma}\right)\right] .
$$

By the similar way, we deduce that

$$
\left\|F_{2}\left(U_{1}\right)-F_{2}\left(U_{2}\right)\right\|_{G_{\sigma, s}^{\delta^{\prime}}} \leq \frac{C_{s}\left\|U_{1}-U_{2}\right\|_{\delta}\left(\left\|U_{0}\right\|_{1}+R\right)}{\left(\delta-\delta^{\prime}\right)^{\sigma}}\left[C_{s}\left(\left\|U_{0}\right\|_{1}+R\right)\left(\frac{9}{2}+\frac{9 e^{-\sigma} \sigma^{\sigma}}{4}\right)+\left(2+e^{-\sigma} \sigma^{\sigma}\right)\right],
$$




$$
\left\|F_{3}\left(U_{1}\right)-F_{3}\left(U_{2}\right)\right\|_{G_{\sigma, s}^{\delta^{\prime}}} \leq \frac{C_{s}\left\|U_{1}-U_{2}\right\|_{\delta}\left(\left\|U_{0}\right\|_{1}+R\right)}{\left(\delta-\delta^{\prime}\right)^{\sigma}}\left[C_{s}\left(\left\|U_{0}\right\|_{1}+R\right)\left(9+\frac{9 e^{-\sigma} \sigma^{\sigma}}{4}\right)+\left(\frac{5}{2}+e^{-\sigma} \sigma^{\sigma}\right)\right],
$$

which lead to

$$
\left\|F\left(U_{1}\right)-F\left(U_{2}\right)\right\|_{\delta^{\prime}} \leq \frac{C_{s}\left\|U_{1}-U_{2}\right\|_{\delta}\left(\left\|U_{0}\right\|_{1}+R\right)}{\left(\delta-\delta^{\prime}\right)^{\sigma}}\left[C_{s}\left(\left\|U_{0}\right\|_{1}+R\right)\left(\frac{45}{2}+\frac{27 e^{-\sigma} \sigma^{\sigma}}{4}\right)+\left(7+3 e^{-\sigma} \sigma^{\sigma}\right)\right] .
$$

The above inequality implies that $F$ satisfies the condition (2) of Theorem 3.1 and $L=C_{s}\left(\left\|U_{0}\right\|_{1}+\right.$ $R)\left[C_{s}\left(\left\|U_{0}\right\|_{1}+R\right)\left(\frac{45}{2}+\frac{27 e^{-\sigma} \sigma^{\sigma}}{4}\right)+\left(7+3 e^{-\sigma} \sigma^{\sigma}\right)\right]$. Moreover $T_{0}=\min \left\{\frac{1}{2^{2 \sigma+4} L}, \frac{\left(2^{\sigma}-1\right) R}{\left(2^{\sigma}-1\right) 2^{2 \sigma+3} L R+M}\right\}$, by setting $R=\left\|U_{0}\right\|_{1}$, we see that $L=C_{s}^{2}\left\|U_{0}\right\|_{1}^{2} C_{1, \sigma}+C_{s}\left\|U_{0}\right\|_{1} C_{2, \sigma}, M \leq 2^{2 \sigma+3} L R$. Then, we deduce that $T_{0}=\frac{1}{2^{2 \sigma+4} L}=\frac{1}{2^{2 \sigma+4}\left[C_{s}^{2}\left\|U_{0}\right\|_{1}^{2} C_{1, \sigma}+C_{s}\left\|U_{0}\right\|_{1} C_{2, \sigma}\right]}$ where $C_{1, \sigma}=90+27 e^{-\sigma} \sigma^{\sigma}$ and $C_{2, \sigma}=14+6 e^{-\sigma} \sigma^{\sigma}$.

\section{Continuity of the data-to-solution map}

In this section, we investigate the continuity of the data-to-solution map for initial data and solutions in Theorems 4.1, 4.2 and 4.4 We only prove this for the 3-component Camassa-Holm system (3CH) since it is much complex and the proofs are similar for the other systems.

Theorem 5.1. Let $\sigma \geq 1$ and $s>\frac{1}{2}$. Assume that $\left(u_{0}, v_{0}, w_{0}\right) \in\left(G_{\sigma, s}^{1}(\mathbb{R})\right)^{3}$. Then the data-to-solution map $\left(u_{0}, v_{0}, w_{0}\right) \mapsto(u, v, w)$ of the 3-component Camassa-Holm system is continuous from $\left(G_{\sigma, s}^{1}(\mathbb{R})\right)^{3}$ into the solutions space.

Firstly we introduce a definition to explain what means the data-to-solution map is continuous from $\left(G_{\sigma, s}^{1}(\mathbb{R})\right)^{3}$ into the solutions space.

Definition 5.2. Let $\sigma \geq 1$ and $s>\frac{1}{2}$. We say that the data-to-solution map $\left(u_{0}, v_{0}, w_{0}\right) \mapsto(u, v, w)$ of the 3-component Camassa-Holm system is continuous if for a given $\left(u_{0}^{\infty}, v_{0}^{\infty}, w_{0}^{\infty}\right) \in\left(G_{\sigma, s}^{1}(\mathbb{R})\right)^{3}$ there exists a $T=T\left(\left\|u_{0}^{\infty}\right\|_{G_{\sigma, s}^{1}},\left\|v_{0}^{\infty}\right\|_{G_{\sigma, s}^{1}},\left\|w_{0}^{\infty}\right\|_{G_{\sigma, s}^{1}}\right)>0$ such that for any sequence $\left(u_{0}^{n}, v_{0}^{n}, w_{0}^{n}\right) \in$ $\left(G_{\sigma, s}^{1}(\mathbb{R})\right)^{3}$ and $\left\|u_{0}^{n}-u_{0}^{\infty}\right\|_{G_{\sigma, s}^{1}}+\left\|v_{0}^{n}-v_{0}^{\infty}\right\|_{G_{\sigma, s}^{1}}+\left\|w_{0}^{n}-w_{0}^{\infty}\right\|_{G_{\sigma, s}^{1}} \stackrel{n \rightarrow \infty}{\longrightarrow} 0$, the corresponding solutions $\left(u^{n}, v^{n}, w^{n}\right)$ of (3CH) satisfy $\left\|u^{n}-u^{\infty}\right\|_{E_{T}}+\left\|v^{n}-v^{\infty}\right\|_{E_{T}}+\left\|w^{n}-w^{\infty}\right\|_{E_{T}} \stackrel{n \rightarrow \infty}{\longrightarrow} 0$, where

$$
\|u\|_{E_{T}}=\sup _{|t|<\frac{T(1-\delta)^{\sigma}}{2^{\sigma}-1}, 0<\delta<1}\left(\|u(t)\|_{G_{\sigma, s}^{\delta}}(1-\delta)^{\sigma} \sqrt{1-\frac{|t|}{T(1-\delta)^{\sigma}}}\right) .
$$

Proof of Theorem 5.1. Without loss of generality, we may assume that $t \geq 0$. As in the proof of Theorem 4.4, we use the same notation $U^{n}=\left(u^{n}, v^{n}, w^{n}\right)^{\mathrm{T}}, U_{0}^{n}=\left(u_{0}^{n}, v_{0}^{n}, w_{0}^{n}\right)^{\mathrm{T}}$ and $\left\|U^{n}\right\|_{\delta}=$ $\left\|u^{n}\right\|_{G_{\sigma, s}^{\delta}}+\left\|v^{n}\right\|_{G_{\sigma, s}^{\delta}}+\left\|w^{n}\right\|_{G_{\sigma, s}^{\delta}}$. Define that

$$
T^{\infty}=\frac{1}{2^{2 \sigma+4}\left[C_{s}^{2}\left\|U_{0}^{\infty}\right\|_{1}^{2} C_{1, \sigma}+C_{s}\left\|U_{0}^{\infty}\right\|_{1} C_{2, \sigma}\right]}, \quad T^{n}=\frac{1}{2^{2 \sigma+4}\left[C_{s}^{2}\left\|U_{0}^{n}\right\|_{1}^{2} C_{1, \sigma}+C_{s}\left\|U_{0}^{n}\right\|_{1} C_{2, \sigma}\right]},
$$


where $C_{1, \sigma}=90+27 e^{-\sigma} \sigma^{\sigma}, C_{2, \sigma}=14+6 e^{-\sigma} \sigma^{\sigma}$ and $C_{s}$ is given in Proposition 2.5 Since $\| U_{0}^{n}-$ $U_{0}^{\infty} \|_{1} \stackrel{n \rightarrow \infty}{\longrightarrow} 0$, it follows that there exists a constant $N$ such that, if $n \geq N$ we have

$$
\left\|U_{0}^{n}\right\|_{1} \leq\left\|U_{0}^{\infty}\right\|_{1}+1
$$

By setting

$$
T=\frac{1}{2^{2 \sigma+4}\left[C_{s}^{2}\left(\left\|U_{0}^{\infty}\right\|_{1}+1\right)^{2} C_{1, \sigma}+C_{s}\left(\left\|U_{0}^{\infty}\right\|_{1}+1\right) C_{2, \sigma}\right]},
$$

we deduce from (5.2) that $T<\min \left\{T^{n}, T^{\infty}\right\}$ for any $n \geq N$. As in the proof of Theorem 4.4 we see that $T^{n}$ and $T^{\infty}$ are the existence time corresponding to $\left\|U_{0}^{n}\right\|_{1}$ and $\left\|U_{0}^{\infty}\right\|$ respectively, which implies that for any $n \geq N$

$$
\begin{aligned}
& U^{\infty}(t, x)=U_{0}^{\infty}(x)+\int_{0}^{t} F\left(U^{\infty}(t, x)\right) d \tau, \quad 0 \leq t<\frac{T(1-\delta)^{\sigma}}{\left(2^{\sigma}-1\right)}, \\
& U^{n}(t, x)=U_{0}^{n}(x)+\int_{0}^{t} F\left(U^{n}(t, x)\right) d \tau, \quad 0 \leq t<\frac{T(1-\delta)^{\sigma}}{\left(2^{\sigma}-1\right)}
\end{aligned}
$$

where $F$ is given in (4.11). From the above equations, we verify that for any $0 \leq t<\frac{T(1-\delta)^{\sigma}}{\left(2^{\sigma}-1\right)}$ and $0<\delta<1$

$$
\left\|U^{n}(t)-U^{\infty}(t)\right\|_{\delta} \leq\left\|U_{0}^{\infty}-U_{0}^{n}\right\|_{\delta}+\int_{0}^{t}\left\|F\left(U^{n}(\tau)\right)-F\left(U^{\infty}(\tau)\right)\right\|_{\delta} d \tau
$$

Define that $\delta(\tau)=\frac{1}{2}(1+\delta)+\left(\frac{1}{2}\right)^{2+\frac{1}{\sigma}}\left\{\left[(1-\delta)^{\sigma}-\frac{t}{T}\right]^{\frac{1}{\sigma}}-\left[(1-\delta)^{\sigma}+\left(2^{\sigma+1}-1\right) \frac{t}{T}\right]^{\frac{1}{\sigma}}\right\}$. By virtue of Lemma 3.7 we see that $\delta<\delta(\tau)<1$. Taking advantage of (4.30), we obtain $\left\|F\left(U^{n}(\tau)\right)-F\left(U^{\infty}(\tau)\right)\right\|_{\delta} \leq$ $\frac{L\left\|U^{n}(t)-U^{\infty}(t)\right\|_{\delta(\tau)}}{(\delta(\tau)-\delta)^{\sigma}}$ where $L=C_{s}^{2}\left\|U_{0}\right\|_{1}^{2} C_{1, \sigma}+C_{s}\left\|U_{0}\right\|_{1} C_{2, \sigma}$. Plugging it into (5.7) yields that

$$
\left\|U^{n}(t)-U^{\infty}(t)\right\|_{\delta} \leq\left\|U_{0}^{\infty}-U_{0}^{n}\right\|_{\delta}+L \int_{0}^{t} \frac{\left\|U^{n}(t)-U^{\infty}(t)\right\|_{\delta(\tau)}}{(\delta(\tau)-\delta)^{\sigma}} d \tau .
$$

Applying Lemma 3.7 with $a=T$, we deduce that

$$
\left\|U^{n}(t)-U^{\infty}(t)\right\|_{\delta} \leq\left\|U_{0}^{\infty}-U_{0}^{n}\right\|_{\delta}+L \frac{T 2^{2 \sigma+3}\left\|U^{n}-U^{\infty}\right\|_{E_{T}}}{(1-\delta)^{\sigma}} \sqrt{\frac{T(1-\delta)^{\sigma}}{T(1-\delta)^{\sigma}-t}} .
$$

Since $T=\frac{1}{2^{2 \sigma+4}\left[C_{s}^{2}\left(\left\|U_{0}^{\infty}\right\|_{1}+1\right)^{2} C_{1, \sigma}+C_{s}\left(\left\|U_{0}^{\infty}\right\|_{1}+1\right) C_{2, \sigma}\right]}$ and $L=C_{s}^{2}\left\|U_{0}\right\|_{1}^{2} C_{1, \sigma}+C_{s}\left\|U_{0}\right\|_{1} C_{2, \sigma}$, it follows that $L T 2^{2 \sigma+3}<\frac{1}{2}$. Then, we have

$$
\left\|U^{n}(t)-U^{\infty}(t)\right\|_{\delta} \leq\left\|U_{0}^{\infty}-U_{0}^{n}\right\|_{\delta}+\frac{1}{2(1-\delta)^{\sigma}}\left\|U^{n}-U^{\infty}\right\|_{E_{T}} \sqrt{\frac{T(1-\delta)^{\sigma}}{T(1-\delta)^{\sigma}-t}},
$$

which leads to

$$
\left\|U^{n}(t)-U^{\infty}(t)\right\|_{\delta}(1-\delta)^{\sigma} \sqrt{1-\frac{t}{T(1-\delta)^{\sigma}}} \leq\left\|U_{0}^{\infty}-U_{0}^{n}\right\|_{\delta}(1-\delta)^{\sigma} \sqrt{1-\frac{t}{T(1-\delta)^{\sigma}}}+\frac{1}{2}\left\|U^{n}-U^{\infty}\right\|_{E_{T}}
$$




$$
\leq\left\|U_{0}^{\infty}-U_{0}^{n}\right\|_{1}+\frac{1}{2}\left\|U^{n}-U^{\infty}\right\|_{E_{T}} .
$$

Note that the right hand side of the above inequality is independent of $t$ and $\delta$. By taking the supremum over $0<\delta<1,0<t<\frac{T(1-\delta)^{\sigma}}{2^{\sigma}-1}$, we obtain that

$$
\left\|U^{n}-U^{\infty}\right\|_{E_{T}} \leq\left\|U_{0}^{\infty}-U_{0}^{n}\right\|_{1}+\frac{1}{2}\left\|U^{n}-U^{\infty}\right\|_{E_{T}}
$$

which implies that

$$
\left\|U^{n}-U^{\infty}\right\|_{E_{T}} \leq 2\left\|U_{0}^{\infty}-U_{0}^{n}\right\|_{1}
$$

The above inequality holds true for any $n \geq N$ and leads to our desire result.

Remark 5.3. In the period case, the Sobolev-Gevrey norm can be stated as follows

$$
\|f\|_{G_{\sigma, s}^{\delta}(\mathbb{T})}=\left(\sum_{k \in \mathbb{Z}}\left(1+|k|^{2}\right)^{s} e^{2 \delta|k|^{\frac{1}{\sigma}}}|\widehat{f}(k)|^{2}\right)^{\frac{1}{2}}=\left\|e^{\delta(-\Delta)^{\frac{1}{2 \sigma}}} f\right\|_{H^{s}(\mathbb{T})},
$$

and the similar propositions still hold true. Taking advantage of Theorem 3.1 and by virtue of the same argument as in Theorems 4.1, 4.2, 4.4 and 5.1, we get the similar Gevrey regularity and analytic for the Camassa-Holm type systems.

Acknowledgements. This work was partially supported by NNSFC (No.11271382), RFDP (No. 20120171110014), the Macao Science and Technology Development Fund (No. 098/2013/A3) and the key project of Sun Yat-sen University.

\section{References}

[1] M. S. Baouendi and C. Goulaouic, Remarks on the abstract form of nonlinear Cauchy-Kovalevsky theorems, Communications in Partial Differential Equations, 2(11) (1977), 1151-1162.

[2] M. S. Baouendi and C. Goulaouic, Sharp estimates for analytic pseudodifferential operators and application to Cauchy problems, Journal of Differential Equations, 48 (1983), 241-268.

[3] R. F. Barostichi, A. Himonas and G. Petronilho, Autonomous Ovsyannikov theorem and applications to nonlocal evolution equation and systems, Journal of Functional Analysis, to appear.

[4] A. Bressan and A. Constantin, Global conservative solutions of the Camassa-Holm equation, Archive for Rational Mechanics and Analysis, 183 (2007), 215-239.

[5] A. Bressan and A. Constantin, Global dissipative solutions of the Camassa-Holm equation, Analysis and Applications, 5 (2007), 1-27. 
[6] R. Camassa and D. D. Holm, An integrable shallow water equation with peaked solitons, Physical Review Letters, 71 (1993), 1661-1664.

[7] R. Camassa, D. Holm and J. Hyman, A new integrable shallow water equation, Advances in Applied Mechanics, 31 (1994), 1-33.

[8] A. Constantin, The Hamiltonian structure of the Camassa-Holm equation, Expositiones Mathematicae, 15(1) (1997), 53-85.

[9] A. Constantin, On the scattering problem for the Camassa-Holm equation, Proceedings of The Royal Society of London, Series A, 457 (2001), 953-970.

[10] A. Constantin, Global existence of solutions and breaking waves for a shallow water equation: a geometric approach, Annales de l'Institut Fourier (Grenoble), 50 (2000), 321-362.

[11] A. Constantin, The trajectories of particles in Stokes waves, Inventiones Mathematicae, 166 (2006), 523535.

[12] A. Constantin and J. Escher, Global existence and blow-up for a shallow water equation, Annali della Scuola Normale Superiore di Pisa - Classe di Scienze, 26 (1998), 303-328.

[13] A. Constantin and J. Escher, Well-posedness, global existence, and blowup phenomena for a periodic quasi-linear hyperbolic equation, Communications on Pure and Applied Mathematics, 51 (1998), 475-504.

[14] A. Constantin and J. Escher, Wave breaking for nonlinear nonlocal shallow water equations, Acta Mathematica, 181 (1998), 229-243.

[15] A. Constantin and J. Escher, Particle trajectories in solitary water waves, Bulletin of the American Mathematical Society, 44 (2007), 423-431.

[16] A. Constantin and J. Escher, Analyticity of periodic traveling free surface water waves with vorticity, Annals of Mathematics, 173 (2011), 559-568.

[17] A. Constantin and D. Lannes, The hydrodynamical relevance of the Camassa-Holm and Degasperis-Procesi equations, Archive for Rational Mechanics and Analysis, 192 (2009) 165-186.

[18] A. Constantin and L. Molinet Global weak solutions for a shallow water equation, Communications in Mathematical Physics, 211 (2000), 45-61.

[19] A. Constantin and W. A. Strauss, Stability of peakons, Communications on Pure and Applied Mathematics, 53 (2000), 603-610.

[20] A. Constantin and R. Ivanov, On an integrable two-component Camassa- Holm shallow water system, Physics Letters A, 372 (2008), 7129-7132. 
[21] R. Danchin, A few remarks on the Camassa-Holm equation. Differential Integral Equations, 14 (2001), 953-988.

[22] J. Escher, O. Lechtenfeld and Z. Yin, Well-posedness and blow-up phenomena for the 2-component Camassa-Holm equation, Discrete and Continuous Dynamical Systems - Series A, 19 (2007), 493-513.

[23] C. Foias and R. Temam, Gevrey class regularity for the solutions of the Navier-Stokes equations, Journal of Functional Analysis, 87 (1989), 359-369.

[24] A. Fokas and B. Fuchssteiner, Symplectic structures, their Bäcklund transformation and hereditary symmetries, Physica D, 4(1) (1981/82), 47-66.

[25] C. Guan and Z. Yin, Global existence and blow-up phenomena for an integrable two-component CamassaHolm shallow water system, Journal of Differential Equations, 248 (2010), 2003-2014.

[26] C. Guan, K.H. Karlsen and Z. Yin, Well-posedness and blow-up phenomena for a modified two-component Camassa-Holm equation, Contemporary Mathematics, 526 (2010), 199-220.

[27] C. Guan and Z. Yin, Global weak solutions for a two-component Camassa- Holm shallow water system, Journal of Functional Analysis, 260 (2011), 1132-1154.

[28] C. Guan and Z. Yin, Global weak solutions for a modified two-component Camassa-Holm equation, Annales de Institut Henri Poincare (C) Non Linear Analysis, 28 (2011), 623-641.

[29] X. G. Geng and B. Xue, A three-component generalization of Camassa-Holm equation with N-peakon solutions, Advances in Mathematics, 226 (2011), 827-839.

[30] G. Gui and Y. Liu, On the global existence and wave-breaking criteria for the two-component CamassaHolm system, Journal of Functional Analysis, 258 (2010), 4251-4278.

[31] D. D. Holm, L. Naraigh and C. Tronci, Singular solution of a modified twocomponent Camassa-Holm equation, Physical Review E, 79 (2009), 1-13.

[32] A. Himonas and G. Misio lek, Analyticity of the Cauchy problem for an integrable evolution equation, Mathematische Annalen, 327 (2003) no.3, 575-584.

[33] T. Kato and G. Ponce, Commutator estimates and the Euler and Navier-Stokes equations, Communications on Pure and Applied Mathematics, 41 (1988), 891-907.

[34] Y. Liu and Z. Yin, Global existence and blow-up phenomena for the Degasperis-Procesi equation, Communications in Mathematical Physics, 267 (2006), 801-820.

[35] W. Luo and Z. Yin, Global existence and local well-posedness for a three-component Camassa-Holm system with N-peakon solutions, Journal of Differential Equations, 259 (2015), 201-234. 
[36] L. Nirenberg, An abstract form of the nonlinear Cauchy-Kowalevski theorem, Journal of Differential Geometry, 6 (1972), 561-576.

[37] T. Nishida, A note on a theorem of Nirenberg, Journal of Differential Geometry, 12 (1977), 629-633.

[38] L.V. Ovsyannikov, Singular operators in Banach spaces scales, Doklady Akademii Nauk SSSR, 163 (1965) 819-822.

[39] L.V. Ovsyannikov, Non-local Cauchy problems in fluid dynamics, Actes du Congrès International des Mathématiciens 3 (1970), 137-142.

[40] L.V. Ovsyannikov, A nonlinear Cauchy problem in a scale of Banach spaces, Doklady Akademii Nauk SSSR 200 (1971), 789-792.

[41] G. Rodríguez-Blanco, On the Cauchy problem for the Camassa-Holm equation, Nonlinear Analysis, Theory Methods Application, 46 (2001), 309-327.

[42] W. Tan and Z. Yin, Global conservative solutions of a modified two-component Camassa-Holm shallow water system, Journal of Differential Equations, 251 (2011), 3558-3582.

[43] W. Tan and Z. Yin, Global dissipative solutions of a modified two-component Camassa-Holm shallow water system, Journal of Mathematical Physics, 52 (2011), 033507.

[44] F. Trèves, Ovsyannikov theorem and hyperdifferential operators, Notas de Matemática, 46 (1968), 238 pp.

[45] F. Trèves, An abstract nonlinear Cauchy-Kovalevska theorem, Transactions of the American Mathematical Society, 150 (1970), 77-92.

[46] J. F. Toland, Stokes waves, Topological Methods in Nonlinear Analysis, 7 (1996), 1-48.

[47] Z. Xin and P. Zhang, On the weak solutions to a shallow water equation, Communications on Pure and Applied Mathematics, 53 (2000), 1411-1433.

[48] K. Yan and Z. Yin, Analytic solutions of the Cauchy problem for two-component shallow water systems, Mathematische Zeitschrift 269 (2011), no. 3-4, 1113-1127.

[49] K. Yan and Z. Yin, Well-posedness for a modified two-component Camassa-Holm system in critical spaces, Discrete and Continuous Dynamical Systems - Series A, 33 (2013), 1699-1712. 